\title{
Tensile response of elastoplastic lattices at finite strain
}

\author{
H C Tankasala, V S Deshpande and N A Fleck* \\ Cambridge University Engineering Dept., Trumpington St., Cambridge, CB2 1PZ, U.K., \\ *Corresponding author. Email: naf1@eng.cam.ac.uk
}

13 January 2017

\begin{abstract}
The finite strain, uniaxial tensile response of two-dimensional (2D) elastoplastic lattices is investigated using finite element simulations and analytical models, taking into full account the macroscopic stiffening due to cell wall alignment. Four morphologies of 2D lattice are considered: triangular, Kagome, hexagonal, and diamond. The cell walls are treated as Timoshenko beams made from an elastoplastic solid with a strain hardening characteristic that resembles Ramberg-Osgood at low strains and exponential hardening at large strains. This description captures the response of metallic lattices at small strain and selected polymeric lattices at large strain. The use of beam theory is validated by additional continuum element simulations. The dependence of macroscopic ductility and tensile strength of each lattice is determined as a function of relative density, cell wall rupture strain and cell wall strainhardening. Two failure criteria are invoked: (i) maximum value of local tensile strain anywhere in the lattice attains a pre-defined failure strain, or (ii) maximum value of average tensile strain across any section of the lattice attains the failure strain. The sensitivity of macroscopic ductility and ultimate tensile strength to geometric imperfection is explored by considering: (i) random topologies in which the joints are randomly perturbed in position, and (ii) a finite crack formed by an array of broken cell walls. The notion of a transition flaw size for the lattices is validated by means of a notch sensitivity analysis, and the significance of crack-tip blunting by cell wall alignment is highlighted for the hexagonal honeycomb.
\end{abstract}

Keywords: lattice materials, ductility, strain hardening, transition flaw size, finite strain

\section{Introduction}

The existing literature on polymeric and metallic foams and lattices is focused on their in-plane macroscopic compressive and shear response (Papka and Kyriakides, 1994; Grediac, 1993; Wang and McDowell, 2004; Gibson and Ashby, 1997; Côté et al; 2006). Triangular 
and Kagome lattices have high in-plane stiffness and strength compared to hexagonal and diamond lattices for a wide range of loading conditions as they are stretching-dominated rather than bending-dominated (Wang and McDowell, 2004; Fleck and Qiu, 2007; Romijn and Fleck, 2007; Hutchinson and Fleck, 2006). Existing analytical models assume small strains and give useful formulae for the macroscopic modulus and yield strength of these lattices as a function of relative density and topology, but they neither inform the large strain response, nor the ductility and ultimate tensile strength (UTS). For example, it remains to quantify the switch in macroscopic response of an elastomeric hexagonal lattice from a compliant, bendingdominated mode to a stiff, stretching-dominated mode as the cell walls align with the tensile axis under increasing macroscopic strain.

The current study addresses the finite strain in-plane tensile response of four elastoplastic 2D lattices as made by additive manufacture: triangular, Kagome, hexagonal and diamond, as shown in Fig. 1(a)-(d), respectively. The choice of these four lattices is motivated by the broad range in their mechanical behaviours: the hexagonal lattice is a compliant, bending-dominated structure whereas the triangular and Kagome lattices are stiff, stretchingdominated structures. Since the triangular, Kagome, and hexagonal lattices possess $120^{\circ}$ rotational symmetry, they are isotropic in their in-plane linear response, but display anisotropy in their non-linear behaviour. In contrast, the diamond lattice is strongly anisotropic in both its linear elastic and non-linear elastic responses: it is a compliant, bending-dominated structure when sheared along the direction of the cell walls, but is a stiff, stretching structure under direct straining in the cell wall-direction. The dependence of macroscopic ductility and ultimate tensile strength upon relative density, cell wall failure strain and strain-hardening exponent are determined. Each lattice has cell walls of length $\ell_{0}$ and thickness $t_{0}$, such that the relative density $\bar{\rho}$ is given by

$$
\bar{\rho}=A \frac{t_{0}}{\ell_{0}}
$$

with the values of the coefficient $A$ listed in Table 1, see for example Gibson and Ashby (1997) and Fleck and Qiu (2007).

It is widely appreciated that as-manufactured lattices contain defects such as cell wall waviness, non-uniform wall thickness, displaced nodes, and thickened nodes (akin to Plateau borders for the case of foams) as discussed in several studies, see for example Zhu et al (2001), Yang and Huang (2005), Symons and Fleck (2008) and Fleck and Qiu (2007). The thrust of the present paper is to explore the sensitivity of ductility and UTS to topology, and to include 
finite deformations in the analysis. Imperfections are limited to randomly displaced nodes and missing cell walls, as prior studies on the yield of lattices suggest that these are amongst the most potent of imperfections in knocking down the strength, see for example Ronan et al. (2016) for the case of the imperfect hexagonal honeycomb.

\subsection{Review of the effective properties of perfect lattices}

The macroscopic in-plane Young's modulus and tensile strength of an elastic-brittle lattice, and the plastic collapse strength of an elastic-perfectly plastic lattice can be estimated from simple beam theory. Here, we summarise the relevant scaling laws for the effective properties of the lattices of Fig. 1, as taken from the literature.

\section{Elastic modulus}

Consider an elastic-brittle lattice made from a solid of cell wall modulus $E_{S}$. The macroscopic Young's modulus $E$ in the $x_{2}$ direction of each lattice of Fig. 1 scales with $\bar{\rho}$ according to

$$
E=B \bar{\rho}^{b} E_{S}
$$

The values of the constants $(B, b)$ are listed in Table 1 , as taken from Gibson and Ashby (1997), Deshpande et al (2001), and Fleck and Qiu (2007).

\section{Fracture strength of elastic-brittle lattice}

A brittle lattice fails at a macroscopic nominal fracture strength $s_{\mathrm{f}}^{\infty}$ when the maximum local tensile stress within any cell wall in the lattice attains the tensile strength of the solid $\sigma_{\mathrm{f}}$ . The diamond lattice of Fig. 1 has pronounced anisotropy in tensile strength (Romijn and Fleck, 2007), whereas the degree of anisotropy is small for the triangular, hexagonal, and Kagome lattices. For definiteness, we shall limit attention to the tensile fracture strength $s_{\mathrm{f}}^{\infty}$ in the $x_{2}$ direction of each lattice; it scales with $\sigma_{\mathrm{f}}$ and $\bar{\rho}$ according to

$$
s_{\mathrm{f}}^{\infty}=C_{1} \bar{\rho}^{c} \sigma_{\mathrm{f}}
$$

where the values of $C_{1}$ and $c$, as listed in Table 1, have been taken from Gibson and Ashby (1997) and Fleck and Qiu (2007). 
Plastic collapse strength of an elastic-perfectly plastic lattice

The macroscopic plastic collapse strength $s_{Y}$ of a bending-dominated lattice (such as the hexagonal and diamond lattices) is defined by the macroscopic stress corresponding to plastic collapse by rotation of the cell walls about fully plastic hinges of moment $M_{P}=\sigma_{\mathrm{YS}} b t_{0}^{2} / 4$, where $\sigma_{\mathrm{YS}}$ is the yield strength of the elastic, perfectly plastic solid and $b$ is the out-of-plane width of the cell wall. For such 2D lattices, the macroscopic yield strength scales with $\bar{\rho}^{2}$, see Gibson and Ashby (1997). In contrast, the macroscopic yield of the stretching-dominated triangular and Kagome lattices requires cell wall stretching, and $s_{Y}$ scales as $\bar{\rho}$ (Wang and McDowell, 2004). None of the lattices of Fig. 1 has an isotropic yield strength. For example, a $30^{\circ}$ rotation of the triangular and Kagome lattices leads to a $50 \%$ change in the value of $s_{Y}$, independent of $\bar{\rho}$ (Deshpande et al; 2001 and Hutchinson et al; 2003). In broad terms, the plastic collapse strength in the $x_{2}$ direction of each lattice scales with $\sigma_{\mathrm{YS}}$ and $\bar{\rho}$ according to

$$
s_{\mathrm{Y}}=C_{2} \bar{\rho}^{c} \sigma_{\mathrm{YS}}
$$

where $\left(C_{2}, c\right)$ are listed in Table 1, and are taken from Gibson and Ashby (1997), Côté et al (2006), and Wang and McDowell (2004).

\subsection{Topological imperfections}

In practice, lattice materials contain defects such as randomly misplaced joints, missing cell walls, cell walls of variable thickness (such as Plateau borders in foams) or of wavy profile, and spatial variations in relative density. The effect of such imperfections on the mechanical properties of lattices was quantified by Fleck and co-workers ( Fleck and Qiu, 2007, Romijn and Fleck, 2007, Quintana Alonso and Fleck, 2010, and Symons and Fleck, 2008) for the elastic-brittle case. They found that the Kagome and diamond lattices experience severe knockdown in both strength and in-plane modulus due to randomly misplaced joints, whereas the triangular and hexagonal lattices are nearly imperfection-insensitive. The imperfectioninsensitivity of the hexagonal lattice extends to the elastoplastic tensile response: Chen et al. $(1999,2001)$ found that the uniaxial tensile strength is unaffected by the presence of rigid inclusions and holes, consistent with experimental observations on aluminium alloy foams 
(Olurin et al; 2000).

Recently, the role of imperfections of 'horse-shoe' geometry on the tensile response of triangular, Kagome and hexagonal lattices has been explored by Ma et al. (2016). The cell walls are treated as linear elastic, but large macroscopic deformations and large cell wall rotations are included. They exploited the fact that cell wall waviness significantly reduces the axial stiffness of cell walls for the triangular and Kagome lattices: although these lattices are stretchingdominated on the length-scale of the cell wall due to their high nodal connectivity, the waviness of cell walls increases their compliance to membrane-type loading. Thus, the macroscopic lattice is much more compliant than the perfect triangular lattice. In contrast, the hexagonal lattice is bending-dominated at the cell wall level (due to its low nodal connectivity), and the presence of additional cell wall waviness has a much more modest effect. These concepts of structural hierarchy are reviewed in Fleck et al. (2010). The present paper has a somewhat different focus than that of Ma et al. (2016). Our aim here is to explore the sensitivity of ultimate tensile strength (UTS) and ductility to lattice topology for straight cell walls made from a non-linear solid, and to include the role of imperfections in the form of misplaced nodes and of missing cell walls.

In addition to the macroscopic mechanical properties of stiffness and strength, the transition flaw size is an important material property. In broad terms, it is the flaw size at which fracture switches from strength-control to toughness-control. For an elastoplastic solid, we define $a_{\mathrm{Ty}}$ as the semi-length of an internal flaw such that the remote stress associated with the attainment of the fracture toughness $K_{\text {IC }}$ equals the macroscopic yield strength $s_{Y}$ of the lattice in the absence of a crack, giving $a_{\mathrm{Ty}}=K_{\mathrm{IC}}^{2} / \pi s_{\mathrm{Y}}^{2}$ (Sih et al., 1965). Alternatively, for the case of an elastic-brittle lattice, we define the transition flaw size $a_{\mathrm{Tf}}$ as the semi-length of an internal flaw such that the remote stress associated with the attainment of the fracture toughness $K_{\mathrm{IC}}$ equals the macroscopic fracture strength $s_{\mathrm{f}}^{\infty}$ of the lattice in the absence of a crack, $a_{\mathrm{Tf}}=K_{\mathrm{IC}}^{2} / \pi s_{\mathrm{f}}^{\infty 2}$. The magnitude of $a_{\mathrm{Tf}}$ in relation to the cell size $\ell_{0}$ is sensitive to the choice of lattice, as revealed by Fleck and Qiu (2007) for the elasticbrittle case. They found that $a_{\mathrm{Tf}} \approx \ell_{0}$ for the hexagonal and triangular lattices, while $a_{\mathrm{Tf}}=0.056 \ell_{0} / \bar{\rho}$ for the Kagome lattice. In a parallel study, Quintana Alonso and Fleck (2007) found that $a_{\mathrm{Tf}}=0.55 \ell_{0} / \bar{\rho}^{2}$ for the diamond lattice. Thus, the hexagonal and triangular lattices are flaw sensitive, while the Kagome and diamond lattices are much more 
flaw tolerant, particularly at low values of relative density. The predicted dependence of $a_{\mathrm{Tf}}$ upon $\bar{\rho}$ for the diamond lattice was verified by fracture tests on edge-cracked cordierite lattices (see Quintana-Alonso et al; 2010). The current study addresses the degree to which these conclusions extend to ductile lattices at finite strain. We shall obtain explicit expressions for $a_{\mathrm{Ty}}$ for the four lattices, and assess the relevance of this parameter to the transition from strength-control to toughness-control.

\subsection{Scope of study}

The macroscopic uniaxial stress versus strain response is obtained for each of the four lattices of Fig. 1. We assume a constitutive law for the cell wall material that is power law in nature at low strains, in order to model metallic lattices. Such lattices fail when the necking strain is attained in the cell wall, or by microvoid coalescence at precipitates which have segregated to grain boundaries during solidification (Mangipudi and Onck (2012)). We extend our treatment to a new class of engineering copolymers, thermoplastic polyolefin (TPO $)^{1}$, that display a power law response at low strains and exponential hardening at high strains, see for example Poon et al. (2007) and Katbab et al. (2000). Such copolymers find widespread application as cellular solids in lightweight transport applications. The tensile failure strain of these copolymers is dictated by molecular disentanglement rather than by necking.

In our unified treatment we adopt a constitutive law that is power law hardening at low strains and exponential hardening at large strains. We treat the tensile failure strain as an additional material parameter. For metals, we can assume that the failure strain equals the (Considére) necking strain, but this is not enforced in the analysis. The uniaxial true stress $\sigma_{t}$ versus true strain $\varepsilon_{t}$ response is taken to be

$$
\varepsilon_{t}=\frac{\sigma_{t}}{E_{\mathrm{S}}}+\varepsilon^{p}
$$

where $E_{\mathrm{S}}$ is the initial Young's modulus, and the plastic strain $\varepsilon^{p}$ depends upon $\sigma_{t}$ according to

\footnotetext{
${ }^{1}$ Also known as olefinic thermoplastic elastomers, these copolymers comprise a thermoplastic such as polypropylene, an ethylene-based elastomer, and a small fraction of a filler such as carbon fibre or fibre glass, thus having physical properties spanning the range between plastics and elastomers. The elastomeric character of these copolymers stems from physical rather than chemical cross linking (Tse and Jiang, 2013).
} 


$$
\frac{\sigma_{t}}{\sigma_{0 \mathrm{~S}}}=\left(1+\frac{\varepsilon^{p}}{\varepsilon_{0 \mathrm{~S}}}\right)^{\frac{1}{n}} \exp \left(\varepsilon^{p}\right)
$$

Here, $n$ is a strain hardening exponent, $\sigma_{0 \mathrm{~S}}$ is a representative 'yield' strength, $\varepsilon_{0 \mathrm{~S}}$ is a representative yield strain and $E_{\mathrm{S}} \equiv \sigma_{0 \mathrm{~S}} / \varepsilon_{0 \mathrm{~S}}$. We emphasize that this material description is designed to capture the initial non-linear yield-type response of a metallic alloy or an engineering polymer at small strain and the ensuing exponential hardening response at large strain as typified by polyolefin elastomers. Typical stress-strain curves of the form as defined in Eq. (5) and Eq. (6) are plotted in Fig. 2 for selected values of $n$.

The predicted macroscopic ductility and UTS of the elastoplastic lattices are based on the maximum value of either :

(i) the local tensile strain (LTS) at any point in the lattice, $\varepsilon_{M}$, or

(ii) the average tensile strain (ATS) across any cell wall of the lattice, $\varepsilon_{A}$.

Analytical expressions for the dependence of macroscopic nominal stress and cell-wall strain ( $\varepsilon_{M}$ and $\varepsilon_{A}$ ) upon the macroscopic nominal strain are derived from a unit cell analysis; see Appendix A. Throughout this study, the accuracy of the analytical models for the perfect lattices is assessed via finite element (FE) simulations. Additionally, the FE study is used to explore the sensitivity of ductility and UTS to imperfections of the form of: (i) randomly misplaced joints, and (ii) a finite crack as defined by a row of missing cell walls. The transition crack length $a_{\mathrm{Ty}}$ is predicted for the ductile lattice from a knowledge of its macroscopic yield strength and fracture toughness. And finally, the accuracy of the concept of a transition flaw size is evaluated from FE predictions of strength versus crack length for the triangular and hexagonal lattices.

\section{Finite element method for uniaxial tensile response of perfect lattices}

The macroscopic tensile response in the $x_{2}$-direction of each lattice of Fig. 1 is obtained from corresponding periodic unit cell calculations. All calculations have been performed using the finite element (FE) package ABAQUS Standard (version 6.14). We emphasise that the flow theory of plasticity is assumed for a cell wall response given by Eq.(6). Each cell wall (or strut) of the unit cell is represented by up to 150 Timoshenko beam elements (of type B21 in ABAQUS notation); these two-noded linear elements account for both bar 
stretching and bending. The Timoshenko beam elements are also geometrically nonlinear so that the strains and rotations can be arbitrarily large. Periodic boundary conditions are imposed such that the macroscopic nominal strain $e_{i j}^{\infty}$ is specified $^{2}$ via the following constraints:

$$
\Delta u_{1}=e_{11}^{\infty} \Delta x_{1}, \quad \Delta u_{2}=e_{22}^{\infty} \Delta x_{2}, \text { and } \Delta \theta=0
$$

where $\Delta u_{i}$ and $\Delta \theta$ are the displacement and rotation jumps, respectively, between corresponding points on the opposite sides of the unit cell, while $\Delta x_{j}$ is the displacement vector connecting these points. All calculations presented here are for an imposed tensile strain $e^{\infty} \equiv e_{22}^{\infty}$ in the $x_{2}$-direction of the unit cell, with the macroscopic nominal stress $s^{\infty} \equiv s_{22}^{\infty}$ i.e., the work conjugate to $e^{\infty}$ being the outcome of the solution. Natural boundary conditions were imposed in the $x_{1}$-direction such that the nominal stress $s_{11}^{\infty}$ (i.e., the work conjugate to $e_{11}^{\infty}$ ) vanishes. FE simulations are performed in both small strain and finite strain, for comparison purposes. The small strain assumption does not capture the stretching that develops with finite rotation of the struts, but serves to verify the accuracy of the analytical models in the small strain regime.

The accuracy of the non-linear Timoshenko beam elements has been checked by performing selected FE simulations using both Timoshenko beam elements and 2D continuum elements in plane strain. Specifically, each strut in the continuum formulation was idealised by CPE4 quadrilateral elements, with typically 10 elements in the thickness direction and 100 elements along the length. Numerical experimentation confirmed that the Timoshenko beam elements are adequate for prediction of both ductility and UTS, but the comparison is not given explicitly here for the sake of brevity.

\section{Results}

Full finite element solutions are reported for the elastoplastic response of the four lattices as defined in Fig. 1. Analytical expressions are derived in Appendix A for the small strain and finite strain elastoplastic responses, including the effect of large geometry changes.

\footnotetext{
${ }^{2}$ We have set the nominal strain $e_{12}^{\infty}=0$ so that $x_{1}$ and $x_{2}$ remain principal strain axes throughout the history of imposed loading. Thereby, the finite strain deformation of the Lagrangian nominal strain reduces to Eq. (7).
} 
Selected FE results ${ }^{3}$ are reported for the choice of $\bar{\rho}=0.1$, yield strain of the solid $\varepsilon_{0 \mathrm{~S}}=0.001$, and strain hardening exponent $n=10$. We consider each topology in turn.

\subsection{Triangular lattice}

A representative plot of the macroscopic nominal stress $s^{\infty}$ versus macroscopic nominal strain $e^{\infty}$ for a triangular lattice is shown in Fig. 3, with sketches of the deformation modes included. The overall response of the lattice is characterized by three distinct regimes, regimes I through III, as indicated in Fig. 3. There is satisfactory agreement between the predictions of the analytical descriptions of Appendix A.1 and the FE predictions, as follows.

Regime I is the initial linear elastic response, regime II is the plastic collapse response and regime III is the post-lock-up response wherein all struts of the lattice have aligned with the loading direction $x_{2}$. Consider each regime in turn. Note that in the early stage of regime II, the $s^{\infty}$ versus $e^{\infty}$ response is power-law and of slope $1 / n$ (on a log-log scale). Consequently, the transition point $e^{\infty}=e_{\mathrm{Y}}$ between regimes I and II is identified in Fig. 3 by the intersection of straight line fits to regime I (slope of unity) and regime II (of slope $1 / n$ ). The transition point from regime II to regime III is the macroscopic lock-up strain $e^{\infty}=e_{\mathrm{L}}=1$ according to the analytical model of Appendix A.1.

Regime I : Direct insight into the elastic response of the lattice is obtained by considering the pin-jointed parent truss-lattice. The vertical struts (labelled $\mathrm{C}$ in Fig. 1(a)) undergo affine deformation whereas the inclined struts (labelled A and B in Fig. 1(a)) rotate without stretch. Consequently, $B=1 / 3$ and $b=1$ in Eq. (2). Negligible macroscopic additional stiffness is generated by elastic bending of struts A and B when the pin-jointed truss is replaced by the rigid-jointed frame.

Regime II : The FE and analytical models reveal that the vertical strut $\mathrm{C}$ undergoes plastic stretch while the inclined struts $\mathrm{A}$ and $\mathrm{B}$ remain elastic for $e^{\infty}<0.02$ and undergo plastic bending for $e^{\infty}>0.02$. An adequate representation of the response in regime II is achieved

\footnotetext{
${ }^{3}$ Additional simulations have been performed for several values of $n$ in the range of 3 to 20 and several values of $\bar{\rho}$ in the range of 0.025 to 0.1 but these are omitted here for the sake of brevity.
} 
by again considering the pin-jointed version. The nominal stress $s^{\infty}$ versus nominal strain $e^{\infty}$ is given by Eq. (A.6) upon making the transformations from true stress and strains to nominal values. Note that the macroscopic yield strain $e_{Y}$ of the lattice equals that of the solid $\varepsilon_{0 S}$.

Regime III : The FE and analytical models show that the struts A and B align with the struts of type $\mathrm{C}$ at $e^{\infty}=1$ and begin to carry significant axial load. We identify the macroscopic lockup strain of the lattice, as $e^{\infty}=e_{\mathrm{L}}=1$. For $e^{\infty}>e_{\mathrm{L}}$, all the bars in a pin-jointed triangular truss are stretched axially and they contribute to the macroscopic stress $s^{\infty}$. The $s^{\infty}$ versus $e^{\infty}$ response in regime III is adequately given by Eq. (A.10) where $e_{\mathrm{L}}=1$. Strut $\mathrm{C}$ in a pinjointed triangular truss stretches by $\varepsilon_{A}^{(\mathrm{C})}=\ln \left(1+e^{\infty}\right)$ whereas struts $\mathrm{A}$ and $\mathrm{B}$ stretch by $\varepsilon_{A}^{(\mathrm{A})}=\varepsilon_{A}^{(\mathrm{B})}=\ln \left(1+0.5\left(e^{\infty}-e_{\mathrm{L}}\right)\right)$. The modes of deformation of the struts within the triangular lattice during regimes I through III are summarized in Table 2.

\subsection{Kagome lattice}

A pin-jointed truss analysis suffices for an analytical model of the Kagome lattice since it is a stretching-dominated structure, see Appendix A.2. Fig. 4(a) shows the FE and analytical predictions for the macroscopic nominal stress $s^{\infty}$ versus $e^{\infty}$ response of a typical Kagome lattice, with $\bar{\rho}=0.1, \varepsilon_{0 \mathrm{~S}}=0.001$ and $n=10$. Under remote tensile loading in the $x_{2}$ direction of the lattice, the inclined struts A and B carry tension whereas the horizontal strut C is under compression. The FE and analytical models reveal that, at a critical load, strut $\mathrm{C}$ buckles. The post-buckling response of the lattice involves large rotation (i.e., scissoring) of struts A and B at almost constant macroscopic tensile stress. At a sufficiently large value of $e^{\infty}$, struts A and B align with the loading axis and come into contact; the post-lock up response follows, and this involves an increasing axial load on struts A and B, with the fully buckled strut $\mathrm{C}$ playing a negligible role. The regimes of macroscopic response of Kagome lattice are summarised below, with the analytic models of Appendix A.2 included in Fig. 4(a). In broad terms, excellent agreement is noted between the FE and analytical approaches.

Regime I: The uniaxial response of the lattice is characterized by the effective Young's 
modulus of the lattice, as given in Eq. (2) with $B=1 / 3$ and $b=1$, as reported by Hyun and Torquato (2002). The FE and analytical predictions of the average strain $\varepsilon_{A}$ in each strut is plotted in Fig. 4(b) as a function of the macroscopic nominal strain $e^{\infty}$; recall that the response of struts A and B is identical due to symmetry. The FE predictions in Fig. 4 are in good agreement with the analytical predictions despite the fact that the analytical model considers the lattice as a pin-jointed truss whereas the FE simulations are performed for a rigid-jointed frame. Regime I ends when struts $\mathrm{A}$ and $\mathrm{B}$ yield at $e^{\infty}=e_{\mathrm{Y}} \approx 1.5 \varepsilon_{0 \mathrm{~S}}$.

Regime II: The FE simulations predict that at $e^{\infty}>e_{\mathrm{Y}}$ struts A and B stretch plastically. Strut C shortens elastically and then plastically, until it buckles at a critical value of stress $s_{\mathrm{cr}}^{\infty}$, as indicated by $X$ in Fig. 4(a). This marks the end of regime IIa and the start of regime IIb. Until the onset of this instability, the macroscopic nominal stress versus strain response of the lattice is adequately predicted by the response of a pin-jointed Kagome lattice, as given by Eq. (A.22). The average axial strain $\varepsilon_{A}$ across the section of struts $\mathrm{A}$ and $\mathrm{B}$ in regime IIa is given by Eq. (A.21), and this expression is in excellent agreement with the FE prediction, see Fig. 4(b). The small difference between $\varepsilon_{M}$ and $\varepsilon_{A}$ in the FE prediction is attributed to slight bending of struts $\mathrm{A}$ and $\mathrm{B}$, but this has little effect on the macroscopic response.

At a critical nominal stress $s_{\mathrm{cr}}^{\infty}$ strut $\mathrm{C}$ buckles and regime IIb (the post-buckling regime) ensues. FE simulations confirm that $s_{\mathrm{cr}}^{\infty}$ at the onset of buckling is adequately given by the Engesser-Shanley formula (Engesser, 1889), such that Eq. (A.25) is satisfied. The Kagome lattice collapses transversely in a concertina-like manner ${ }^{4}$ at an almost constant macroscopic tensile stress $\bar{S}^{\infty}$ involving buckling of struts of type C and bending of struts A and B. Eventually, struts A and B align with the loading direction $x_{2}$ at a nominal lock-up strain $e_{\mathrm{L}}=0.42$ and regime III begins. We proceed to use a Maxwell-type construction to obtain the constant tensile collapse stress $\bar{s}^{\infty}$ in regime IIb, from the instant of buckling to the instant of lock-up.

\footnotetext{
${ }^{4}$ The FE post-buckling response of the Kagome lattice is obtained by allowing for inertia stabilization in an explicit dynamic FE analysis. A small loading rate was employed to ensure only a small dynamic contribution and thus the FE response can be considered to be approximately quasi-static.
} 


\section{Maxwell construction of steady-state broadening of crush band}

We idealise the strut $C$ by a simply-supported bar of thickness $t_{0}$, length $\ell_{0}$, and width $b$ under a compressive axial load $F$ and constrained against end transverse displacement, as shown in Fig. 5(a). The bar shortens by $u$ at each end due to $F$. FE simulations of the load versus shortening response are shown in Figs. 5(b)-(c), assuming the material description given by Eq. (6) with $n=5,10$ and 20, and stockiness $t_{0} / \ell_{0}=0.05,0.1$ and 0.15 . After the axial load $F$ attains the Engesser-Shanley buckling load, it drops with increasing shortening until contact of the two ends of the beam-column occurs: this contact condition defines the lockedup state $\left(u / \ell_{0}=u_{\mathrm{L}} / \ell_{0}=0.5-t_{0} / \ell_{0}\right)$. The average collapse load $\bar{F}$ is defined by

$$
\bar{F}=\frac{1}{u_{\mathrm{L}}-u_{1}} \int_{u_{1}}^{u_{\mathrm{L}}} F(u) d u
$$

where $u_{1}$ is such that $F\left(u_{1}\right)=F\left(u_{\mathrm{L}}\right)=\bar{F}$. A scaling law for $\bar{F}$ can be obtained by assuming that the collapse mode is adequately represented by the rotation of an effective plastic hinge at its mid-length. The bar is subdivided into two cantilever beams, each of length $\ell_{0} / 2$ , and subjected to a transverse end force $P$. The work increment $d W_{\mathrm{P}}$ in each of the subbeams is given by $d W_{\mathrm{P}}=P(\delta) d \delta$ where $\delta$ is the transverse end deflection under the load $P$. Now make use of the formula (A.36) for the transverse end deflection $\delta$ of a built-in beam of length $\ell_{0} / 2$ under a transverse end load $P$. The work increment $d W_{\mathrm{P}}$ can be rephrased in terms of an effective moment $M=P \ell_{0} / 2$ and hinge rotation $\omega=2 \delta / \ell_{0}$, such that $M d \omega=P d \delta$. The work done in the bar equals that associated with the rotation of hinges in each of the sub-beams by $\pi / 2$ such that $u_{\mathrm{L}}-u_{1} \approx \ell_{0} / 2$. Consequently, we obtain

$$
W_{\mathrm{P}}=2 \int_{0}^{\pi / 2} M\left(\omega^{\prime}\right) d \omega^{\prime}=Q(n) \frac{\sigma_{0 \mathrm{~S}}}{\left(\varepsilon_{0 \mathrm{~S}}\right)^{1 / n}} b t_{0}\left(\frac{t_{0}}{\ell_{0}}\right)^{\frac{n+1}{n}} \ell_{0}
$$

where the constant of proportionality $Q$ scales only with $n$. Also,

$$
W_{\mathrm{P}}=2 \bar{F}\left(u_{\mathrm{L}}-u_{1}\right) \approx \bar{F} \ell_{0}
$$

and so we obtain

$$
\bar{F}=Q(n) \frac{\sigma_{0 \mathrm{~S}}}{\left(\varepsilon_{0 \mathrm{~S}}\right)^{1 / n}} b t_{0}\left(\frac{t_{0}}{\ell_{0}}\right)^{\frac{n+1}{n}}
$$


We obtain an accurate expression for $Q(n)$ via the FE simulations by making use of Eq. (8) rather than the approximate beam collapse calculation above, as our intent is only to determine the desired scaling relation via the approximate calculation. Numerical evaluation of the integral in Eq. (8) gives the constant of proportionality $Q(n)$; its magnitude is plotted in Fig. 5(d) for selected values of $n$ between 5 and 20 .

Now, the average macroscopic stress $\bar{s}^{\infty}$ associated with the end-to-end shortening of struts $\mathrm{C}$ of the Kagome lattice from $u=u_{1}$ to $u=u_{\mathrm{L}}$ is calculated via a simple work calculation for the unit cell of Fig. 1(b). As the struts $\mathrm{C}$ shorten, the inclined struts A and B scissor and the lattice extends by an axial nominal strain $\Delta e^{\infty}$, such that

$$
\bar{s}^{\infty} \Delta e^{\infty} V_{0}=8 \bar{F}\left(u_{\mathrm{L}}-u_{1}\right)
$$

Here, $V_{0}=4 \sqrt{3} b \ell_{0}^{2}$ is the volume of the unit cell and the factor of 8 on the RHS of Eq. (12) arises because the unit cell contains four horizontal struts, each of width $b$ and length $\ell_{0}$ and each of which shortens by $2\left(u_{\mathrm{L}}-u_{1}\right)$. The magnitude of $\Delta e^{\infty}$ from Fig. $4(\mathrm{a})$ is the increment in nominal strain over regime IIb. Alternatively, the value $\Delta e^{\infty}=0.155$ can be estimated by the nominal macroscopic strain that brings the inclined struts $\mathrm{A}$ and $\mathrm{B}$ of a pin-jointed truss into contact, upon shortening the horizontal strut $\mathrm{C}$ to zero length. The work statement Eq. (12) gives

$$
\bar{s}^{\infty}=\frac{R(n)}{\left(\varepsilon_{0 \mathrm{~S}}\right)^{1 / n}} \bar{\rho}^{\frac{2 n+1}{n}} \sigma_{0 \mathrm{~S}}
$$

upon using $\bar{\rho}=\sqrt{3} t_{0} / \ell_{0}$ and upon defining $R(n)=1.24 Q(n) / 3^{1 / 2 n}$. The values of the constant $R(n)$ are plotted in Fig. 5(d) for selected values of $n$ between 5 and 20. Fig. 4(a) shows adequate agreement between the formula Eq. (13) and the FE prediction.

We note in passing that plastic collapse in regime II can alternatively occur by rupture of strut $A$ ( or $B$ ) when the average tensile strain in this strut attains a critical value prior to the onset of buckling of strut $\mathrm{C}$. Assume that rupture occurs when the average tensile strain achieves the value $\varepsilon^{(\mathrm{A})}=1 / n$, thereby identifying it with the Considére necking strain for a power-law hardening solid. The macroscopic nominal strain for the onset of rupture $e_{\mathrm{r}}^{\infty}$ then follows from Eq. (A.21) as 


$$
e_{\mathrm{r}}^{\infty}=\frac{1}{3 n}\left(\frac{1+2^{n+2}}{2^{n}}\right)
$$

The macroscopic strain $e_{\mathrm{cr}}^{\infty}$ associated with the activation of the dominant mode of plastic instability (rupture of struts A or B versus buckling of strut C) is plotted in Fig. 6(a) as a function of $n$ for selected values of $t_{0} / \ell_{0}$ in the range of 0.025 to 0.2 . The switch between modes is identified by equating Eq. (A.26) and Eq. (14) to give

$$
\bar{\rho}=\frac{3}{\pi 2^{n / 2}}
$$

Selected contours of $e_{\mathrm{cr}}^{\infty}$ are plotted as a function of $(\bar{\rho}, n)$ in Fig. 6(b), with the boundary, Eq. (15), included in the map.

Regime III: The FE and analytical models suggest that at $e^{\infty}>e_{\mathrm{L}}=0.42$, the inclined struts of type A and B stretch almost uniformly under the applied strain as given by Eq. (A.28) and the nominal stress-strain response in regime III is adequately given by Eq. (A.29). The modes of deformation within different struts of a Kagome lattice during regimes I through III are summarized in Table 2.

\subsection{Equivalence of the Kagome and triangular lattices}

The above analysis in Section 3.2 suggests that the Kagome lattice undergoes transverse buckling under remote tension, in contrast to the triangular lattice which does not. In fact, both lattices are anisotropic in their plastic response and we shall now explain that the transverse tensile response of the Kagome lattice (along $x_{1}$-direction) of Fig. 1(b) is almost identical to the longitudinal tensile response of the triangular lattice (along $x_{2}$-direction), as defined in Fig. 1(a). Likewise, the transverse tensile response of the triangular lattice (loaded along $x_{1}$ direction) is very close to that of the Kagome lattice loaded along the $x_{2}$-direction.

Transverse tension of the Kagome lattice: The struts of type $\mathrm{C}$ undergo uniaxial tension when the Kagome lattice is subjected to transverse tension along the $x_{1}$-direction. Negligible axial loads exist in the inclined struts of type A and B. Consequently, the analytical model for the triangular lattice, as given by equations (A.3), (A.6), and (A.10), are valid for transverse tension of the Kagome lattice. 
Transverse tension of the triangular lattice: Transverse tension of the triangular lattice induces tension in the inclined struts $\mathrm{A}$ and $\mathrm{B}$ and compression in the vertical strut $\mathrm{C}$ of Fig. 1(a). The analytical models for regimes I, IIa and III (but not IIb) are identical to those already developed for tensile loading of the Kagome lattice along the $x_{2}$-direction, see equations (A.16), (A.22), and (A.29). The critical macroscopic stress $s_{\mathrm{cr}}^{\infty}$ at the onset of buckling in strut C, and the average tensile stress $\bar{s}^{\infty}$ in the transverse direction during the buckling of strut $\mathrm{C}$ in regime IIb can again be determined by the Engesser-Shanley estimate, and by a Maxwell-type construction, respectively. Recall that the Engesser-Shanley buckling load in Eq. (A.23) and the average buckling load $\bar{F}$ in Eq. (11) are expressed in terms of $t_{0} / \ell_{0}$ for a single strut. Consequently, for the triangular lattice, the analytical form for $s_{\mathrm{cr}}^{\infty}$ in Eq. (A.25) and $\bar{s}^{\infty}$ in Eq. (13) is modified to ${ }^{5}$

$$
s_{\mathrm{cr}}^{\infty}=\left(\frac{\pi^{2}}{36 n \varepsilon_{0 \mathrm{~S}}}\right)^{\frac{1}{n}} \frac{n+2}{\rho} \frac{n+}{n} \sigma_{0 \mathrm{~S}}
$$

and

$$
\bar{s}^{\infty}=\frac{T(n)}{\left(\varepsilon_{0 \mathrm{~S}}\right)^{1 / n}} \bar{\rho}^{\frac{2 n+1}{n}} \sigma_{0 \mathrm{~S}}
$$

with $\bar{\rho}=2 \sqrt{3} t_{0} / \ell_{0}, T(n)=0.62 Q(n) /(2 \sqrt{3})^{1 / n}$ and $Q(n)$ already given in Fig. 5(d).

\subsection{Hexagonal lattice}

The macroscopic nominal stress $s^{\infty}$ versus nominal strain $e^{\infty}$ response of an elastoplastic hexagonal lattice is plotted in Fig. 7(a), for the choice of $\bar{\rho}=0.1, \varepsilon_{0 \mathrm{~S}}=0.001$ and $n=10$. Fig. 1(c) identifies the 3 types of struts present in the unit cell of a hexagonal lattice: inclined struts $\mathrm{A}$ and $\mathrm{B}$ oriented at $\pm 60^{\circ}$ to the loading axis $x_{2}$ and vertical strut $\mathrm{C}$ aligned with the loading axis. The various regimes of tensile response of hexagonal lattice are now discussed in turn.

\footnotetext{
${ }^{5}$ Note that the magnitude of the parameter $A$ in Eq. (1) differs for the triangular and Kagome lattices, and the unit cell of the triangular lattice in the work expression Eq. (12) consists of 1 strut of length $\ell_{0}$ giving $V_{0}=$ $\sqrt{3} b \ell_{0}^{2} / 2$, see Fig. 1(a).
} 
Regime I: The linear elastic response of the lattice is characterized by an effective Young's modulus, as given by Eq. (2), with $B=3 / 2$ and $b=3$, see Gibson and Ashby (1997). In regime I, struts A and B bend elastically, with a point of inflection at mid-length. Strut $\mathrm{C}$ is aligned with the loading axis and it stretches elastically. The analytical model for the hexagonal lattice (in Appendix A.3) employs simple beam theory ${ }^{6}$ for struts A and B. It predicts that the maximum local tensile strain $\varepsilon_{M}$ in each strut occurs at the joint, and its magnitude is given by Eq. (A.35). The FE prediction for $\varepsilon_{M}$ in struts $\mathrm{A}$ and B is shown in Fig. 7(b): it is in excellent agreement with the analytical prediction of Eq. (A.35).

Regime II: The macroscopic strain $e^{\infty}$ is almost entirely due to plastic bending of struts A and $B$ with the vertical strut $C$ stretching elastically, see Fig. 7(b). The small strain analytical predictions as given by Eq. (A.38) for the nominal response and Eq. (A.39) for the maximum cell wall strain $\varepsilon_{M}$ in struts $\mathrm{A}$ and $\mathrm{B}$, are adequate in the initial stage of regime II up to $e^{\infty}=0.1$. Thereafter, finite deflection effects stiffen the lattice. It is noted that the value of $\varepsilon_{M}$ as obtained from beam theory (Eq. A.39) suggests that $\varepsilon_{M}$ becomes unbounded as $n \rightarrow \infty$; a continuum analysis indicates that $\varepsilon_{M}$ is finite for the elastic, ideally plastic solid, as discussed by Calladine and English (1984). With increasing macroscopic strain, struts A and $\mathrm{B}$ rotate to align with the tensile direction $x_{2}$ and geometric-hardening of the lattice ensues, see Fig. 7(a). All struts within the lattice are aligned with the loading axis at a macroscopic lock-up strain $e_{\mathrm{L}}=0.33$.

Regime III: The analytical model of Appendix A.3 suggests that the increment in macroscopic strain post lock-up ( $e^{\infty}>e_{\mathrm{L}}=0.33$ ) is due to uniform plastic stretch of strut $\mathrm{C}$ with negligible axial strain accumulation in struts $\mathrm{A}$ and $\mathrm{B}$. This is due to the fact that each strut $\mathrm{C}$ is tied to a pair of struts $\mathrm{A}$ and $\mathrm{B}$, and so it carries twice the axial load. The FE simulation supports the analytical prediction Eq. (A.40) that the axial strain in strut C is uniform and consequently good agreement between the FE and analytical models is achieved for both the macroscopic

\footnotetext{
6 Consider a cantilever beam subjected to a concentrated load at the tip. The tip deflection obtained via Timoshenko beam theory is higher than that obtained with simple beam theory by a factor $1+0.6\left(t_{0} / \ell_{0}\right)^{2}$ (Timoshenko \& Gere 1961). For $\bar{\rho}=0.1$, the aspect ratio of strut $\mathrm{B}$ is $t_{0} / \ell_{0} \approx 1 / 6$ and the two theories almost coincide.
} 
stress $s^{\infty}$ and the axial strain across the section $\varepsilon_{A}$ as a function of $e^{\infty}$, see Figs. 7(a) and (b). There remains one subtlety. The FE simulations reveal a local strain concentration near the joints for struts A and B. This is indicated in Fig. 7(b) by the feature that $\varepsilon_{A}$ for struts A and $\mathrm{B}$ exceeds that for strut $\mathrm{C}$ in regime III. The local strain concentration arises from a combination of finite geometry changes at the joint and the high assumed value for $n$. A summary of the various modes of deformation within the struts of a hexagonal lattice during regimes I through III is provided in Table 2.

\subsection{Diamond lattice}

The struts of a diamond lattice bend in a similar fashion to struts A and B of the hexagonal lattice when the lattice is subjected to uniaxial tension along the $x_{2}$-direction. Consequently, the overall $s^{\infty}$ versus $e^{\infty}$ response of the diamond lattice is qualitatively similar to that of the hexagonal lattice, see Fig. 8. The initial response of the diamond lattice is by elastic bending of the struts, followed by plastic bending until all the struts of the lattice align with the loading axis at a macroscopic nominal lock-up strain $e_{\mathrm{L}}=0.41$. The post lock-up response of the lattice involves plastic stretching of the aligned struts. The three regimes of behaviour of the diamond lattice are now summarised.

Regime I involves elastic bending of each strut. The analytical model for strut A of the hexagonal lattice suffices to capture the response of each strut of the diamond lattice, upon suitably re-expressing the included angle $\alpha$ of the struts, and the relative density $\bar{\rho}$ in terms of $t_{0}$ and $\ell_{0}$ for the diamond lattice, as discussed in Appendix A.3. The effective Young's modulus of the lattice is given by Eq. (2), with $B=1 / 4$ and $b=3$, see for example Romijn and Fleck (2007). The linear elastic response from the FE simulation is in excellent agreement with the analytical expression, see Fig. 8. Likewise, the FE prediction for the maximum value $\varepsilon_{M}$ of the local tensile strain in each strut is accurately predicted by simple beam theory, as given by Eq. (A.35) (not shown here).

Regime II: Each strut of the lattice bends plastically; the lattice stiffens due to a reduction in the moment arm on each strut as a consequence of the alignment of the struts with the loading direction. For $e^{\infty}<0.1$, we find from FE simulations that $\varepsilon_{A} \square \varepsilon_{M}$ so that the macroscopic 
$s^{\infty}$ versus $e^{\infty}$ response is adequately represented by the inextensional beam theory prediction Eq. (A.38). The analytical model under-predicts the $s^{\infty}$ versus $e^{\infty}$ response for $e^{\infty}>0.1$ when the role of finite rotations becomes significant, as seen from Fig. 8 . The struts align with the loading axis at a macroscopic lock-up strain $e^{\infty}=e_{\mathrm{L}}=0.41$.

Regime III: Post lock-up, the struts stretch almost uniformly, with bending adjacent to the joints. The analytical model for this regime is presented in Appendix A.3, and the macroscopic nominal stress versus strain response is given by Eq. (A.45). Again, there is good agreement between the analytical and FE predictions.

\section{The macroscopic ductility and ultimate tensile strength (UTS) of each lattice}

We proceed to assess the ductility and tensile strength of each lattice, and define failure by a local strain criterion. Recall that, for the beam elements adopted here, the distribution of strain is linear across the thickness with an average tensile value $\varepsilon_{A}$ and a maximum tensile value $\varepsilon_{M}$ on the outermost fibre. Two criteria are employed, the local tensile strain (LTS) criterion and the average tensile strain (ATS) criterion, as follows.

LTS criterion: the maximum value $\varepsilon_{M}$ of the local tensile strain anywhere in the lattice attains the failure strain $\varepsilon_{\mathrm{f}}$. Ceramics and brittle metallic alloys such as high strength aluminium alloys develop cracks (for example by shear localisation) when a maximum tensile strain is achieved, and the LTS criterion is appropriate, see for example Onck et al. (2004); Mangipudi and Onck (2011); and Mangipudi and Onck (2012).

ATS criterion: the maximum value $\varepsilon_{A}$ of the average tensile strain across any section of the lattice attains the failure strain $\varepsilon_{\mathrm{f}}$. de Kruijf et al. (2009) have adopted this viewpoint in the context of beams made from highly ductile solids under combined bending and stretching.

In the following subsections, comparisons are made for the macroscopic ductility and tensile strength of elastoplastic lattices with $\bar{\rho}=0.1, \varepsilon_{0 S}=0.001$ and $n=10$. The various lattice topologies listed in Fig. 1 are now compared in terms of their uniaxial tensile response, ductility, and ultimate tensile strength to determine the best choice of topology for structural load bearing applications. By way of introduction, Fig. 9(a) compares the nominal stress 
versus nominal strain response of the four lattices: they are strikingly different in response and so log-log scales are required to display all 4 curves.

\subsection{Ductility}

The lattice ductility $e_{\mathrm{f}}^{\infty}$ is defined as the remote value of strain at which $\varepsilon_{M}$ or $\varepsilon_{A}$ within a critical cell wall attains the failure strain $\varepsilon_{\mathrm{f}}$. Figures 9(b) and 9(c) show the predicted ductility for each lattice for the LTS $\left(\varepsilon_{M}=\varepsilon_{\mathrm{f}}\right)$ and $\operatorname{ATS}\left(\varepsilon_{A}=\varepsilon_{\mathrm{f}}\right)$ criteria, respectively.

\section{Predicted ductility according to the LTS criterion}

The relative ranking of lattice in terms of macroscopic ductility $e_{\mathrm{f}}^{\infty}$ is sensitive to the assumed value of $\varepsilon_{\mathrm{f}}$. For example, for the choice $\varepsilon_{M}=\varepsilon_{\mathrm{f}}=0.1 \%$ we note from Fig. 9(a) that $e_{\mathrm{f}}^{\infty}=0.1 \%, 0.15 \%, 0.22 \%$, and $0.67 \%$ for the triangular, Kagome, hexagonal, and diamond lattices, respectively. In contrast, for $\varepsilon_{\mathrm{f}}=10 \%$, we find that $e_{\mathrm{f}}^{\infty}=5.5 \%, 6.7 \%, 10 \%$, and $16.5 \%$ for the hexagonal, Kagome, triangular, and diamond, respectively. The analytical predictions, as taken from Appendix A for the macroscopic ductility of the four lattices, are of the form ${ }^{7}$

$$
e_{\mathrm{f}}^{\infty}=D \bar{\rho}^{d} \varepsilon_{\mathrm{f}}
$$

where $D=D(n)$, and $d$ is a constant independent of $n$. These predictions are based on the assumption that failure precedes lock-up. The values of $(D, d)$ for each lattice are summarised in Table 3. The analytical predictions of Eq. (18) are included in Fig. 9(b) as dashed lines and exhibit excellent agreement with the FE predictions.

\section{Predicted ductility according to the ATS criterion}

The ductility of triangular and Kagome lattices is insensitive to the choice of ATS versus LTS criteria as the cell walls predominantly stretch, compare Fig. 9(b) and 9(c). In contrast, the

\footnotetext{
7 These are given by Eq. (A.3) for the triangular lattice upon assuming $e_{\mathrm{f}}^{\infty} \square$ 1, Eq. (A.21) for the Kagome lattice, and Eq. (A.39) for hexagonal and diamond lattices.
} 
ductility of the bending-dominated hexagonal and diamond lattices is sensitive to the choice of local (LTS) or cell thickness-average (ATS) failure strain since $\varepsilon_{M} \square \varepsilon_{A}$ in regime II prior to lock-up, and $\varepsilon_{M} \approx \varepsilon_{A}$ in regime III. Also, for the hexagonal and diamond lattices, the analytical models of Appendix A reveal that the level of average tensile strain across the cell wall $\varepsilon_{A}$ is small in the plastic collapse regime II, typically below $1 \%$ in accordance with Eq. (A.39). However, after lock-up $\varepsilon_{A}$ increases in proportion to $e^{\infty}$, as demanded by Eq. (A.40) for the hexagonal lattice and Eq. (A.43) for the diamond lattice. Consequently, for a wide range of cell wall failure strain $\varepsilon_{\mathrm{f}}$ in the range $0.1 \%$ to $10 \%$, the ATS criterion suggests that the macroscopic ductility is close to the lock-up strain (on the order of 30\%-40\%). We shall show that this feature disappears in the presence of large imperfections such as randomly displaced cell walls.

\subsection{Ultimate tensile strength (UTS)}

The ultimate tensile strength (UTS) of each lattice $s_{\mathrm{f}}^{\infty}$ is defined as the value of nominal stress $s^{\infty}$ at which a critical cell wall anywhere in the lattice attains the cell wall failure strain $\varepsilon_{\mathrm{f}}$. A comparison of the UTS of the four lattices is given in Fig. 9(d) for $\bar{\rho}=0.1$ , $\varepsilon_{0 \mathrm{~S}}=0.001$ and $n=10$, for both the LTS $\left(\varepsilon_{M}=\varepsilon_{\mathrm{f}}\right)$ and $\operatorname{ATS}\left(\varepsilon_{A}=\varepsilon_{\mathrm{f}}\right)$ criteria. The following deductions are drawn from Fig. 9(d):

(i) Regardless of the choice of failure criterion, the Kagome lattice has the highest value of UTS, followed by the triangular lattice. The diamond lattice is the next strongest provided $\varepsilon_{\mathrm{f}}$ is less than 0.4 , otherwise the hexagonal lattice is the next strongest.

(ii) The UTS, $s_{\mathrm{f}}^{\infty}$, of the triangular and Kagome lattices is insensitive to the choice of the failure criterion. (The curves for the ATS and LTS criteria overlap each other for the triangular lattice in Fig. 9(d).) Failure occurs in regime I for $\varepsilon_{\mathrm{f}}<0.1 \%$, in regime II for $0.1 \%<\varepsilon_{\mathrm{f}}<60 \%$ and in regime III for $\varepsilon_{\mathrm{f}}>60 \%$. The slope of the log-log plot of $s_{\mathrm{f}}^{\infty}$ versus $\varepsilon_{\mathrm{f}}$ in regime II is close to $1 / n=0.1$, as predicted by the analytical formulae ${ }^{8}$ of Appendix A, and restated here

\footnotetext{
${ }^{8}$ These are obtained by combining Eq. (A.3) and Eq. (A.6) for the triangular lattice, Eq. (A.21) and Eq. (A.22) for Kagome lattice, and Eq. (A.38) and Eq. (A.39) for hexagonal and diamond lattices.
} 


$$
s_{\mathrm{f}}^{\infty}=C \bar{\rho}^{c}\left(\frac{\varepsilon_{\mathrm{f}}}{\varepsilon_{0 \mathrm{~S}}}\right)^{\frac{1}{n}} \sigma_{0 \mathrm{~S}}
$$

where the values of $(C, c)$ are listed in Table 3. This analytical prediction is not displayed in Fig. 9(d), however the quantitative agreement is within 5\% for both lattices, provided failure is within regime II. In regime III, the UTS of the triangular and Kagome lattices scales as

$$
s_{\mathrm{f}}^{\infty}=M \bar{\rho}\left(\frac{\varepsilon_{\mathrm{f}}}{\varepsilon_{0 \mathrm{~S}}}\right)^{\frac{1}{n}} \sigma_{0 \mathrm{~S}}
$$

upon making use of Eq. (A.8) and Eq. (A.10) for the triangular lattice and Eq. (A.28) and Eq. (A.29) for the Kagome lattice. The constant $M$ equals $1 / 3$ for the triangular lattice and equals $1 / \sqrt{3}$ for the Kagome lattice.

(iii) In contrast, the value of $s_{\mathrm{f}}^{\infty}$ for the hexagonal and diamond lattices is extremely sensitive to the choice of the failure criterion. First assume that the ATS criterion holds. Then, as discussed above in the context of macroscopic ductility of these lattices, failure occurs soon after lock-up (regime III) for a wide range of values of cell wall failure strain $\varepsilon_{\mathrm{f}}$. In regime III, the ultimate tensile strength of the hexagonal and diamond lattices, according to the ATS criterion, are adequately given by the analytical predictions of Appendix A.3, upon combining Eqs. (A.40) and (A.42), and Eqs. (A.43) and (A.45) to give Eq. (A.20), where $M=1 / 2$ for the hexagonal lattice and $M=1 / \sqrt{2}$ for the diamond lattice. Second, consider the LTS criterion. Then, failure occurs predominantly in regime II, and the analytical prediction Eq. (19) again applies, with suitable values for $(C, c)$ included in Table 3. Again, the accuracy is adequate for our purposes (to within 5\%), although the comparison is not shown explicitly in the figure.

\section{Effect of imperfections on lattice ductility}

Consider again the perfect 2D lattice topologies as shown in Fig. 1. Random lattices are generated by displacing each node within the perfect lattice to a new random location ${ }^{9}$ (of

\footnotetext{
${ }^{9}$ The random movement of joints increases the average length of each strut, and in turn the relative density $\bar{\rho}$. Romijn \& Fleck (2007) show from a simple geometric construction that the correction factor for $\bar{\rho}$ is between 1.0025 for $R / \ell_{0}=0.1$ and 1.0625 for $R / \ell_{0}=0.5$.
} 
equal probability) to any spot on a circular disc of radius $R$. Examples of imperfect topologies with $R / \ell_{0}=0.5$ are shown in Fig. 10 in their initial unstressed configuration. A series of up to 10 structural realisations has been generated for each lattice, upon making the choice $\bar{\rho}=0.05, \varepsilon_{0 \mathrm{~S}}=0.001$ and $n=10$. We define the lattice ductility $e_{\mathrm{f}}^{\infty}$ as the value of macroscopic nominal strain $e^{\infty}$ at which $\varepsilon_{M}$ or $\varepsilon_{A}$ attain the cell wall failure strain $\varepsilon_{\mathrm{f}}$. The ductility of each lattice of Fig. 10 is determined from a representative volume element of $10 \times 10$ cells subjected to uniaxial tensile loading along the global $x_{2}$-direction. Following the definitions of LTS and ATS criteria in Section 4, we shall assume that the LTS criterion is satisfied at relatively small value of failure strain $\varepsilon_{\mathrm{f}}$ such that $\varepsilon_{M}=\varepsilon_{\mathrm{f}}=0.02$ in the examples that follow; the choice of failure strain $\varepsilon_{\mathrm{f}}$ for the ATS criterion is taken as $\varepsilon_{A}=\varepsilon_{\mathrm{f}}=0.1$ consistent with Considére cell-wall necking at $n=10$.

Consider first the LTS criterion. The mean value of $e_{\mathrm{f}}^{\infty}$ of the imperfect lattice over the 10 realisations is plotted in Fig. 11(a) for selected values of $R / \ell_{0}$ between 0 and 0.5 , and failure in accordance with the LTS criterion. For all lattice topologies, the random movement of nodes leads to a significant knockdown in ductility: the triangular, diamond and hexagonal lattice drop in ductility by a factor of about 4 , while the ductility of the Kagome lattice drops by an order of magnitude with increasing $R / \ell_{0}$. The difference in response for the Kagome lattice is consistent with the previous observation that the modulus, tensile strength and fracture toughness of the elastic-brittle Kagome lattice is the most sensitive to imperfection, recall Romijn and Fleck (2007) .

Second, consider the ATS criterion, see Fig. 11(b). All lattices fail when the struts are deforming in a stretching-mode. This is subsequent to a bending phase of deformation for the hexagonal and diamond lattices, thus they exhibit the highest ductility regardless of the level of imperfection. The hexagonal lattice drops in ductility by a factor of about 2 , whereas the diamond lattice displays a drop by a factor of about 8 when $R / \ell_{0}$ is increased from 0 to 0.5 . Insight into the difference in response is obtained by plotting in Fig. 12 the deformed mesh for these 2 lattices at peak macroscopic stress, for the choice $R / \ell_{0}=0.5$. Each plot includes contours of the average axial stress $\sigma_{A}$ across the cell wall of these 2 lattices, after suitable 
normalisation by $\sigma_{0 \mathrm{~S}}$. Force chains ${ }^{10}$ develop in the hexagonal lattice, such that only a small fraction of the struts carry the macroscopic tensile stress, see Fig. 12(a). In contrast, the diamond lattice in Fig. 12(b) does not develop force chains, rather it exhibits random strain concentrations due to the imperfections; the Kagome and triangular lattice also behave in this manner, with similar knockdowns in ductility with increasing imperfection.

\section{Effect of a finite crack upon tensile strength}

Recall the Dugdale analysis for yield versus fracture of an infinite panel made from an elastic-ideally plastic solid of yield strength $s_{\mathrm{Y}}$ and fracture toughness $K_{\mathrm{IC}}$. The panel contains a finite crack of length $2 a$; then, the panel yields at $s^{\infty}=s_{\mathrm{Y}}$ for a short crack and fractures at $K=K_{\mathrm{IC}}$ for a long crack, see for example Anderson (1995). Now introduce a transition flaw size $a_{\mathrm{Ty}}=K_{\mathrm{IC}}^{2} / \pi s_{\mathrm{Y}}^{2}$ to mark the transition from macroscopic yield to fracture, and define the gross failure stress of the panel by $s_{\mathrm{f}}^{\infty}$. Then, the Dugdale analysis suggests that the dependence of $s_{\mathrm{f}}^{\infty} / s_{\mathrm{Y}}$ upon $a / a_{\mathrm{T}}$ is given by (Anderson 1995)

$$
\frac{a}{a_{\mathrm{Ty}}} \ln \left[\sec \left(\frac{\pi s_{\mathrm{f}}^{\infty}}{2 s_{\mathrm{Y}}}\right)\right]=\frac{\pi^{2}}{8}
$$

Equation (21) is commonly used as the basis of elastoplastic fracture mechanics, and is encapsulated in design codes such as R6 (2001). In the current study, we proceed to explore the extent to which Eq. (21) holds for the case of elastoplastic lattices.

Recall from Fleck and Qiu (2007) and Quintana Alonso and Fleck (2007) that the transition between strength-controlled fracture and toughness-controlled fractures occurs at a small transition crack length $a_{\mathrm{Tf}} \approx \ell_{0}$ for the triangular and hexagonal elastic-brittle lattices. In contrast, the transition crack length is large for the elastic-brittle Kagome lattice, $a_{\mathrm{Tf}} \approx \ell_{0} / \bar{\rho}$, and is very large for the diamond lattice, $a_{\mathrm{Tf}} \approx \ell_{0} / \bar{\rho}^{2}$. Now, we know from Tankasala et al. (2015) that the fracture toughness $K_{\mathrm{IC}}$ of an elastoplastic lattice much exceeds that of the elastic-brittle lattice. Consequently, much larger FE meshes are needed to explore the transition behaviour of the elastoplastic lattice than the elastic-brittle lattice, and

\footnotetext{
10 The formation of force chains is a well-known phenomenon in granular media. The force network inside a granular packing is heterogeneous, with certain chains carrying most of the load (Hidalgo et al., 2004).
} 
we restrict our attention to a centre-cracked panel (CCP) made from triangular and hexagonal elastoplastic lattices.

The CCP contains a macroscopic crack of length $2 a$ in the form of a row of broken cell walls in a perfect lattice of size $2 W \times 2 H$, see Fig. 13(a). The assumed crack morphologies for the triangular and hexagonal lattices are depicted in Fig. 13(b) and Fig. 13(c) respectively. We consider the case $H / W=1.2$ and two extreme values $a / W=0.05$ and 0.5 . The choice $a / W=0.05$ depicts a crack in a large panel but has the shortcoming that very large FE meshes are needed, particularly at large $a / \ell_{0}$. In contrast, the choice $a / W=0.5$ requires relatively small finite element meshes but has the limitation that the panel contains only a few cells across its width at small $a / \ell_{0}$.

The CCP is subjected to remote tensile stress $s^{\infty}$ in the $x_{2}$-direction of the lattice and we write $s^{\infty}=s_{\mathrm{f}}^{\infty}$ at failure. The sensitivity of $s_{\mathrm{f}}^{\infty} / s_{\mathrm{Y}}$ to crack length $a / \ell_{0}$ for the triangular lattice is given in Fig. 14(a) and (c), for the LTS and ATS criteria, respectively. Likewise, the sensitivity of $s_{\mathrm{f}}^{\infty} / s_{\mathrm{Y}}$ to crack length $a / \ell_{0}$ for the hexagonal lattice is given in Fig. 14(b) and (d), for the LTS and ATS criteria, respectively. As before, the cell wall strain to failure $\varepsilon_{\mathrm{f}}$ is taken as $\varepsilon_{\mathrm{f}}=0.02$ for the LTS criterion and $\varepsilon_{\mathrm{f}}=0.1$ for the ATS criterion. For both lattices and for both failure criteria, we assume the representative values $\bar{\rho}=0.05$, $\varepsilon_{0 S}=0.001$ and $n=10$. The analytical Dugdale solution Eq. (21) is included in dashed lines in Fig. 14 for comparison ${ }^{11}$.

It is instructive to include the value of the transition flaw size $a_{\mathrm{Ty}}=K_{\mathrm{IC}}^{2} / \pi s_{\mathrm{Y}}^{2}$ on the abscissa of each plot of Fig. 14, upon taking the value of $s_{\mathrm{Y}}$ from Eq. (4) and Table 1, and $K_{\text {IC }}$ (for both the ATS and LTS criteria) from Tankasala et al. (2015). The reason for adding the value of $a_{\mathrm{Ty}} / \ell_{0}$ to each graph of Fig. 14 is to assess whether the transition from strength to toughness control occurs at $a \approx a_{\mathrm{Ty}}$. The transition crack length $a_{\mathrm{Ty}} / \ell_{0}$ for the LTS criterion is

$$
\frac{a_{\mathrm{Ty}}}{\ell_{0}}=H \bar{\rho}^{h}\left(\frac{\varepsilon_{\mathrm{f}}}{\varepsilon_{0 \mathrm{~S}}}\right)^{\frac{n+1}{n}}
$$

\footnotetext{
${ }^{11}$ The Dugdale expression Eq. (21) is modified in Fig. 14 to include the effect of finite geometry via the geometric calibration factor $Y(a / W, H / W)$, as given in Liu (1996).
} 
with the values of the constants $H(n)$ and $h$ given in Table 4 for both lattices for selected values of $n$. (Values of $(H, h)$ for Kagome and diamond lattices have also been included in Table 4 for completeness). For the ATS criterion, the dependence of $K_{\text {IC }}$ upon $\left(\bar{\rho}, n, \varepsilon_{\mathrm{f}}, \varepsilon_{0 \mathrm{~S}}, \sigma_{0 \mathrm{~S}}, \ell_{0}\right)$ has the same functional form for the ATS and LTS criteria for the triangular lattice, and consequently $a_{\mathrm{Ty}} / \ell_{0}$ is still given by Eq. (22). In contrast, the predicted fracture toughness of the hexagonal lattice is significantly greater for the ATS criterion than for the LTS criterion, as discussed by Tankasala et al. (2015), and $a_{\mathrm{Ty}} / \ell_{0}$ is now given by

$$
\frac{a_{\mathrm{Ty}}}{\ell_{0}} \approx 250\left(\frac{\varepsilon_{\mathrm{f}}}{\varepsilon_{0 \mathrm{~S}}}\right)^{\frac{2}{n}}
$$

It is clear from the 4 plots of Fig. 14 that the strength of the cracked lattice does undergo a transition from strength-control to fracture-control at a crack length equal to $a_{\mathrm{Ty}}$ for both lattices and for both criteria ATS and LTS.

The previous study by Tankasala et al. (2015) found that the magnitude of $a_{\mathrm{Ty}}$ in Eq. (22) and Eq. (23) is comparable to the plastic zone size $r_{\mathrm{P}}$ at the tip of a semi-infinite crack at the onset of failure. Also recall that the usual criterion for the validity of Linear Elastic Fracture Mechanics (LEFM) is that the crack length satisfies $a \geq 2.5\left(K_{\mathrm{IC}} / s_{\mathrm{Y}}\right)^{2} \approx 8 r_{\mathrm{P}}$ for a fully dense solid in plane stress ${ }^{12}$, see for example, Anderson (1995). Thus, a useful measure for the validity of LEFM for a lattice material can be taken to be $a \geq 8 a_{\mathrm{Ty}}$, and this criterion has been added to the abscissa of each plot in Fig. 14. Upon taking the 4 graphs together in Fig. 14 it is clear that the lattice fails in accordance with LEFM when the crack length criterion $a \geq 8 a_{\mathrm{Ty}}$ is met. In this regime, $s_{\mathrm{f}}^{\infty}$ scales with $a^{-1 / 2}$ as expected for the long crack case.

\subsection{Sensitivity of strength to crack length for triangular lattice}

Fig. 14(a) shows the notch sensitivity of the triangular lattice for $a / W=0.05$ and $a / \ell_{0}$ in the range of 1 to 10 , for the case of the LTS criterion. (Prohibitively large FE meshes

\footnotetext{
${ }^{12}$ Although the plastic zone size directly ahead of the crack tip in plane strain is about a third that of the zone size in plane stress, the extent of the plastic zone from the crack tip, off-line of the crack is comparable for plane stress and plane strain.
} 
are needed for longer crack lengths.) The notch strength slightly exceeds the yield strength of a perfectly-plastic triangular lattice due to the low value of strain-hardening considered here $(n=10)$. The transition crack length is $a_{\mathrm{Ty}} / \ell_{0} \approx 18$ (for the choice $\varepsilon_{\mathrm{f}}=0.02$ ) via Eq. (22), implying that LEFM is valid for $a \geq 8 a_{\mathrm{Ty}} \approx 144 \ell_{0}$. It was impractical to perform finite element simulations in the LEFM regime for $a / W=0.05$ due to the prohibitively large mesh size. Instead, the notch response in the LEFM regime was explored by performing FE calculations on the crack geometry $a / W=0.5$; these are included in Fig. 14(a). We find from in Fig. 14(a) that the notch tensile strength of the triangular lattice asymptotes to the LEFM prediction for $a \geq 8 a_{\mathrm{Ty}}$. Similar observations are made for the ATS criterion as shown in Fig. 14(c). The transition flaw size is then $a_{\mathrm{Ty}} / \ell_{0} \approx 106$ (for $\varepsilon_{\mathrm{f}}=0.1$ ), and the FE predictions indicate that the notch response is adequately given by the LEFM estimate for $a \geq 8 a_{\mathrm{Ty}} \approx 848 \ell_{0}$.

\subsection{Sensitivity of strength to crack length for the hexagonal lattice}

The notch sensitivity of the hexagonal lattice is plotted in Fig. 14(b) according to LTS criterion. The transition flaw size is $a_{\mathrm{Ty}} / \ell_{0} \approx 9$ such that, for $a<a_{\mathrm{Ty}}$, fracture is strengthcontrolled, whereas for $a \geq 8 a_{\mathrm{Ty}} \approx 72 \ell_{0}$ LEFM conditions prevail. Again, the notch strength in the strength-controlled regime is slightly higher than the yield strength of a perfectly plastic hexagonal lattice as a result of minor strain hardening in the cell wall material. Consider now the ATS criterion. The transition flaw size for $\varepsilon_{\mathrm{f}}=0.1$ is $a_{\mathrm{Ty}} / \ell_{0} \approx 454$ according to Eq.

(23). We note from Fig. 14(d) that the notch strength $s_{\mathrm{f}}^{\infty}$ for $a / \ell_{0}<70$ is up to an order of magnitude higher than the yield strength $s_{\mathrm{Y}}$ of the perfectly plastic hexagonal lattice as a result of geometric hardening of the lattice: cells wall align with the loading axis.

\subsection{The elastic-brittle case}

The notch sensitivity curves for the elastic-brittle triangular and hexagonal lattices are plotted in Fig. 15(a) and (b). Failure occurs when the local maximum tensile stress $\sigma_{M}$ attains the failure strength $\sigma_{\mathrm{f}}$ of the solid: this is a LTS-type of criterion but based upon stress and not strain. The strength of the pre-cracked lattice is plotted as $s_{\mathrm{f}}^{\infty}(a) / s_{\mathrm{f}}^{\infty}(0)$ versus 
$a / \ell_{0}$, and the transition flaw size is defined as $a_{\mathrm{Tf}}=\frac{1}{\pi}\left(K_{\mathrm{IC}} / s_{\mathrm{f}}^{\infty}(a=0)\right)^{2}$. Again, the minimum crack length for which LEFM applies is taken to be $8 a_{\mathrm{Tf}}$. The Dugdale prediction Eq. (21) now becomes

$$
\frac{a}{a_{\mathrm{Tf}}} \ln \left[\sec \left(\frac{\pi s_{\mathrm{f}}^{\infty}}{2 s_{\mathrm{f}}^{\infty}(a=0)}\right)\right]=\frac{\pi^{2} Y^{2}}{8}
$$

where the Dugdale expression has been modified by to include the effect of finite geometry on the $K$-calibration via the geometric calibration factor $Y(a / W, H / W)$, as given in Liu (1996). The analytical prediction (24) has been added to the 2 plots of Fig. 15, and is accurate over the full range of $a / \ell_{0}$ for the triangular lattice, see Fig. 15(a), and in the LEFM regime $a>8 a_{\mathrm{Tf}}$ for the hexagonal lattice. Equation (24) is slightly non-conservative, however, for crack lengths shorter than $8 a_{\mathrm{Tf}}$ in the hexagonal lattice, see Fig. 15(b).

\section{Concluding remarks}

The uniaxial tensile response of 2D elastoplastic lattices has been analysed for 4 morphologies: triangular, Kagome, hexagonal, and diamond lattices. We have demonstrated that the tensile of each lattice is characterized by 3 regimes of behaviour: (i) initial linear elastic regime, (ii) plastic collapse regime as a result of cell wall bending or stretching, and (iii) postlock up stretching-dominated regime due to the rotation of the inclined struts towards the macroscopic loading direction. The analytical models adequately capture the lattice response in all 3 regimes. Additionally, when the Kagome or triangular lattice is so-orientated with regard to the macroscopic loading direction that it has transverse struts, a plastic buckling mechanism is activated. Buckling of these transverse struts leads to the formation of crush bands aligned with the macroscopic direction of loading. The macroscopic tensile stress is almost constant in the phase of crush band broadening; this has been captured by a simple Maxwell-like construction for the post-buckling collapse response of a single transverse strut.

Our study reveals that the macroscopic ductility and ultimate tensile strength of perfect 2D elastoplastic lattices depends on the relative density, the extent of strain-hardening in the strut material, cell wall failure strain and the choice of the failure criterion. We find that the stretching-dominated triangular and Kagome lattices each have comparable tensile strengths for the LTS and ATS criteria. In contrast, the macroscopic strength of the bending-dominated hexagonal and diamond lattices drop significantly when the ATS criterion is replaced by the 


\section{LTS criterion.}

The role of imperfections in the form of randomly misplaced joints on the macroscopic ductility of the lattice has been assessed for the 4 elastoplastic lattices. For the LTS failure criterion, we find that that the ductility of the Kagome lattice drops by an order of magnitude with increasing degree of randomness, consistent with the previously observed sensitivity of elastic-brittle Kagome lattice to the random movement of nodes, recall Romijn and Fleck (2007). For the ATS failure criterion, the hexagonal lattice has the highest ductility regardless of the level of imperfection.

Finally, the notion of a transition flaw size has been analysed for the elastoplastic triangular and hexagonal lattices; their elastic-brittle counterparts are extremely flaw sensitive, with the transition crack length on the order of the cell size. The transition flaw size is sensitive to the assumed failure strain: the transition flaw size increases dramatically with increasing failure strain, as made explicit by Eqs. (22) and (23). We find that the LEFM prediction for notch tensile strength is adequate when the crack length exceeds 8 times the transition crack length. Additionally, it is found that strain hardening in the strut material and the alignment of struts with the macroscopic loading direction increase the tensile strength of the hexagonal lattice in the notch-insensitive regime by up to an order of magnitude above the yield strength of the ideally plastic lattice.

\section{Acknowledgements}

The authors are grateful for financial support of this work in the form of an ERC MULTILAT grant 669764 . 


\section{APPENDIX A: Analytical models for the tensile response of each lattice}

Analytical models are derived herein for each of the four lattices of Fig. 1. The response of each lattice is subdivided into three regimes as follows. When subjected to an increasing macroscopic strain, the lattice responds elastically, regime I, followed by plastic collapse, regime II. At sufficiently large macroscopic strain the cell walls align with the loading direction, termed 'lock-up'. Post lock-up, the cell walls stretch under uniform tension, termed regime III. In order to obtain analytical expressions for the macroscopic response, we shall assume small strains and deformations in regime I (elastic) and in regime II (plastic collapse). This assumption is fully justified for regime I, and holds in the initial stage of regime II up to a macroscopic nominal tensile strain of the lattice on the order of 0.15 . The finite element analysis reveals that regime II persists to much larger values of macroscopic nominal strain, as shown in Figs. 3,4,7 and 8. Regime II ends at the onset of lock-up at finite deformation of each lattice. Thereafter, all lattices behave in a stretching manner, and simple analytical formulae can be established for the post lock-up regime III.

In regime I, the macroscopic response of nominal stress $s^{\infty}$ versus nominal strain $e^{\infty}$ is determined analytically by assuming that the cell wall true stress $\sigma$ versus true strain $\varepsilon$ response is linear and is given by $\sigma=E_{\mathrm{S}} \varepsilon$, where the cell wall Young's modulus is $E_{\mathrm{S}}=$ $\sigma_{0 \mathrm{~S}} / \varepsilon_{0 \mathrm{~S}}$ in terms of the yield strength $\sigma_{0 \mathrm{~S}}$ and yield strain $\varepsilon_{0 \mathrm{~S}}$. The macroscopic response in regimes II and III is estimated by neglecting the linear elastic contribution to Eq. (6), such that, the true stress $\sigma$ versus true strain $\varepsilon$ in the cell wall satisfies

$$
\frac{\sigma}{\sigma_{0 S}}=\exp (\varepsilon)\left(\frac{\varepsilon}{\varepsilon_{0 S}}\right)^{\frac{1}{n}}
$$

where $\sigma_{0 \mathrm{~S}}$ is the yield strength, $\varepsilon_{0 \mathrm{~S}}$ is the yield strain and $n$ is the strain hardening exponent of the solid. The analytical models developed below are plotted in figures 3,4,7 and 8 for the triangular, Kagome, hexagonal, and diamond lattice, respectively. For any given topology, the inclined struts A and B (as labelled in Fig. 1) deform in the same manner. We proceed to derive the analytical models for each lattice, in turn.

\section{A.1. Triangular lattice}

The triangular lattice has a sufficiently high nodal connectivity of six that its macroscopic response is stretching-dominated and is adequately represented by a pin-jointed truss, see for example Deshpande et al. (2001). Write $s^{\infty}$ and $e^{\infty}$ as the macroscopic nominal stress and strain, respectively, and $\sigma^{(\mathrm{C})}$ and $\varepsilon^{(\mathrm{C})}$ as the true measures of stress and strain, 
respectively, in the struts of type $\mathrm{C}$; these struts are aligned with the loading direction $x_{2}$ as indicated in Fig. 1(a). The off-axis struts (A and B) carry no load. Then, in regimes I and II, equilibrium dictates:

$$
\sigma^{(\mathrm{C})}=\frac{\sqrt{3}}{2} \frac{\ell_{0}}{t^{(C)}} s^{\infty}
$$

and the kinematics of the unit cell gives

$$
\varepsilon^{(\mathrm{C})}=\ln \frac{\ell^{(\mathrm{C})}}{\ell_{0}}=\ln \left(1+e^{\infty}\right)
$$

Here, the subscript 0 denotes the value in the reference (undeformed) configuration. Upon recalling that $\bar{\rho}=2 \sqrt{3} t_{0} / \ell_{0}$ and assuming $t \ell=t_{0} \ell_{0}$ by incompressibility, we have from Eq. (A.2) and Eq. (A.3),

$$
\sigma^{(\mathrm{C})}=\frac{3}{\bar{\rho}}\left(1+e^{\infty}\right) s^{\infty}
$$

and the macroscopic response in regime $\mathrm{I}\left(e^{\infty} \ll 1\right)$ is

$$
s^{\infty}=\frac{\bar{\rho}}{3} E_{\mathrm{S}} e^{\infty}
$$

In similar fashion, the macroscopic response in regime II, upon making use of Eq. (A.1), is

$$
\frac{s^{\infty}}{\sigma_{0 S}}=\frac{\bar{\rho}}{3}\left[\frac{\ln \left(1+e^{\infty}\right)}{\varepsilon_{0 S}}\right]^{\frac{1}{n}}
$$

Regime II ends at the point of geometric lock-up of the lattice, such that the inclined struts $\mathrm{A}$ and $\mathrm{B}$ have rotated by $60^{\circ}$ to align with the vertical strut $\mathrm{C}$ and begin to carry axial load. Upon assuming negligible cell wall extension and negligible axial force in the inclined struts $\mathrm{A}$ and $\mathrm{B}$ within regimes I and II, the nominal lock-up strain $e_{\mathrm{L}}$ of the lattice is given by $e_{\mathrm{L}}=1$ by straightforward geometry. Regime III $\left(e^{\infty}>e_{\mathrm{L}}\right)$ ensues, and all struts in the pinjointed parent truss undergo stretching. Within regime III, the true axial strain within the struts reads

$$
\varepsilon^{(\mathrm{C})}=\ln \frac{\ell^{(\mathrm{C})}}{\ell_{0}}=\ln \left(1+e^{\infty}\right)
$$

for strut $\mathrm{C}$, and

$$
\varepsilon^{(\mathrm{A})}=\varepsilon^{(\mathrm{B})}=\ln \frac{\ell^{(A)}}{\ell_{0}}=\ln \left(1+\frac{1}{2}\left(e^{\infty}-e_{\mathrm{L}}\right)\right)
$$

for struts $\mathrm{A}$ and $\mathrm{B}$. Force equilibrium gives

$$
s^{\infty} \frac{\sqrt{3} \ell_{0}}{2}=\sigma^{(\mathrm{C})} t^{(\mathrm{C})}+\sigma^{(\mathrm{A})} t^{(\mathrm{A})}
$$


so that, upon combining relations (A.1) and (A.7) to (A.9), the macroscopic response in regime III reads

$$
\frac{s^{\infty}}{\sigma_{0 S}}=\frac{\bar{\rho}}{3}\left[\frac{1}{\varepsilon_{0 S}} \ln \left(1+e^{\infty}\right)\right]^{\frac{1}{n}}+\frac{\bar{\rho}}{3}\left[\frac{1}{\varepsilon_{0 S}} \ln \left(1+\frac{1}{2}\left(e^{\infty}-e_{\mathrm{L}}\right)\right)\right]^{\frac{1}{n}}
$$

\section{A.2. Kagome lattice}

The Kagome lattice is also a stiff, stretching-dominated structure. Under uniaxial tensile loading of the pin-jointed unit cell in the $x_{2}$ direction, the inclined struts $\mathrm{A}$ and $\mathrm{B}$ are in tension whereas the horizontal strut $\mathrm{C}$ is in compression. Consider first the initial, small deformation response of regime I and the initial stage of regime IIa. Then, equilibrium gives the true axial stresses $\sigma^{(\mathrm{A})}, \sigma^{(\mathrm{B})}$ and $\sigma^{(\mathrm{C})}$ in struts $\mathrm{A}, \mathrm{B}$ and $\mathrm{C}$, respectively, in terms of the nominal stress $s^{\infty}$ as

$$
\sigma^{(\mathrm{A})}=\sigma^{(\mathrm{B})}=\frac{2}{\sqrt{3}} \frac{\ell_{0}}{t^{(\mathrm{A})}} s^{\infty}
$$

and

$$
\sigma^{(\mathrm{C})}=-\frac{1}{\sqrt{3}} \frac{\ell_{0}}{t^{(\mathrm{C})}} s^{\infty}
$$

Compatibility of the unit cell gives the cell wall (true) strain in terms of the deformed lengths of struts $\mathrm{A}$ (and $\mathrm{B}$ ) and $\mathrm{C}, \ell^{(\mathrm{A})}$ and $\ell^{(\mathrm{C})}$, respectively, and the initial length $\ell_{0}$ such that

$$
\exp \left(2 \varepsilon^{(\mathrm{A})}\right)=\frac{1}{4} \exp \left(2 \varepsilon^{(\mathrm{C})}\right)+\frac{3}{4}\left(1+e^{\infty}\right)^{2}
$$

where

$$
\varepsilon^{(\mathrm{A})}=\ln \frac{\ell^{(\mathrm{A})}}{\ell_{0}} \quad \text { and } \quad \varepsilon^{(\mathrm{C})}=\ln \frac{\ell^{(\mathrm{C})}}{\ell_{0}}
$$

Since the strains $\left(\varepsilon^{(\mathrm{A})}, \varepsilon^{(\mathrm{C})}, e^{\infty}\right)$ are small we can simplify Eq. (A.13) to

$$
e^{\infty}=\frac{4}{3} \varepsilon^{(\mathrm{A})}-\frac{1}{3} \varepsilon^{(\mathrm{C})}
$$

Consequently, the macroscopic response in regime $I$ follows from equations (A.11), (A.12), (A.14) and (A.15) as

$$
s^{\infty}=\frac{\bar{\rho}}{3} E_{\mathrm{S}} e^{\infty}
$$

with the axial strains in the struts given by

$$
\varepsilon^{(\mathrm{A})}=\varepsilon^{(\mathrm{B})}=-2 \varepsilon^{(\mathrm{C})}=\frac{2}{3} e^{\infty}
$$

Likewise, the initial response in regime IIa follows by making use of Eq. (A.1) in preference 
to the relation $\sigma=E_{\mathrm{S}} \varepsilon$, as follows. Combine Eq. (A.11), Eq. (A.12) and Eq. (A.14) to obtain the true axial stress in struts $\mathrm{A}$ and $\mathrm{B}$ as

$$
\sigma^{(\mathrm{A})}=\sigma^{(\mathrm{B})}=\frac{2}{\bar{\rho}}\left(\exp \varepsilon^{(\mathrm{A})}\right) s^{\infty}
$$

and in strut $\mathrm{C}$ as

$$
\sigma^{(\mathrm{C})}=-\frac{1}{\bar{\rho}}\left(\exp \varepsilon^{(\mathrm{C})}\right) s^{\infty}
$$

The relation between the true axial stress in struts $\mathrm{B}$ and $\mathrm{C}$ follows by substitution of Eq. (A.18) and Eq.(A.19) into Eq. (A.1), to give

$$
\varepsilon^{(B)}=-2^{n} \varepsilon^{(C)}
$$

Now substitute Eq. (A.20) into Eq. (A.15) to obtain the axial strain in each strut as

$$
\varepsilon^{(\mathrm{A})}=\varepsilon^{(\mathrm{B})}=-2^{n} \varepsilon^{(\mathrm{C})}=3 \frac{2^{n}}{1+2^{n+2}} e^{\infty}
$$

The macroscopic nominal stress in the initial stage of regime IIa follows immediately from Eq. (A.11) to give

$$
\frac{s^{\infty}}{\sigma_{0 S}}=\frac{\bar{\rho}}{2}\left[3\left(\frac{2^{n}}{1+2^{n+2}}\right) \frac{e^{\infty}}{\varepsilon_{0 S}}\right]^{\frac{1}{n}}
$$

\section{Plastic buckling response in regime II}

At a critical nominal stress $S_{\mathrm{cr}}^{\infty}$, strut $\mathrm{C}$ buckles: regime IIa ends and regime IIb (the postbuckling regime) ensues. The buckling load, $P_{\mathrm{cr}}^{(\mathrm{C})}$ for a strut $\mathrm{C}$ of thickness $t_{0}$, length $\ell_{0}$, and width $b$ is given by the Engesser-Shanley formula:

$$
P_{\mathrm{cr}}^{(\mathrm{C})}=\frac{k^{2} \pi^{2} E_{S T} I}{\ell_{0}^{2}}
$$

where the end constraint factor $k$ depends on the degree of constraint to rotation at joints, $E_{\mathrm{ST}}$ is the tangent modulus of the solid, and $I=b t_{0}{ }^{3} / 12$ is the second moment of inertia of the cell wall cross-section. FE simulations performed on an infinite Kagome lattice are discussed in the main body of the paper (see for example Fig. 4) and suggest that $k=2$ is an adequate approximate for the capturing the buckling load at the start of regime IIb. Upon making use of Eq. (A.1), the buckling criterion (A.23) gives the critical strain in strut $\mathrm{C}, \varepsilon_{\mathrm{cr}}^{(\mathrm{C})}$ at the onset of buckling as

$$
\varepsilon_{\mathrm{cr}}^{(\mathrm{C})}=-\frac{\pi^{2}}{9 n} \bar{\rho}^{2}
$$

The corresponding macroscopic nominal stress, $s_{\mathrm{cr}}^{\infty}$ and nominal strain, $e_{\mathrm{cr}}^{\infty}$ follow by 
application of Eq. (A.21) and Eq. (A.22) into Eq. (A.24) as

$$
S_{\mathrm{cr}}^{\infty}=\sigma_{0 S}\left[\frac{\pi^{2}}{9 n \varepsilon_{0 S}}\right]^{\frac{1}{n}} \frac{n+2}{\rho}
$$

and

$$
e_{\mathrm{cr}}^{\infty}=\pi^{2} \frac{\left(1+2^{n+2}\right)}{27 n} \bar{\rho}^{2}
$$

respectively.

The post-buckling response of the Kagome lattice is detailed in the main body of the paper, and a Maxwell construction is used to determine the constant applied macroscopic tensile stress until the point of lock-up. In this post-buckling regime, the struts A and B bend and unload axially, while the horizontal strut $\mathrm{C}$ progressively shortens by buckling. Assume that struts $\mathrm{A}$ and $\mathrm{B}$ rotate as rigid bodies without extension. The lattice locks-up at a macroscopic nominal lock-up strain $e_{\mathrm{L}}$, such that struts $\mathrm{A}$ and $\mathrm{B}$ align with the loading direction. The nominal macroscopic lock-up strain $e_{\mathrm{L}}$ reads

$$
e_{\mathrm{L}}=\frac{2}{\sqrt{3}}\left(1+\varepsilon_{\mathrm{cr}}^{(\mathrm{A})}\right)-1
$$

where $\varepsilon_{\mathrm{cr}}^{(\mathrm{A})}$ is the critical axial strain in strut $\mathrm{A}$ (and B) at the onset of buckling obtained from combining Eq. (A.21) and Eq. (A.24).

Post lock-up, struts A and B undergo affine deformation such that

$$
\varepsilon^{(\mathrm{A})}=\varepsilon^{(\mathrm{B})} \approx \ln \left(1+\varepsilon_{\mathrm{cr}}^{(\mathrm{A})}+\frac{\sqrt{3}}{2}\left(e^{\infty}-e_{\mathrm{L}}\right)\right)
$$

and the macroscopic nominal stress in regime III reads

$$
\frac{s^{\infty}}{\sigma_{0 \mathrm{~S}}}=\frac{\bar{\rho}}{\sqrt{3}}\left[\frac{1}{\varepsilon_{0 \mathrm{~S}}} \ln \left(1+\varepsilon_{\mathrm{cr}}^{(\mathrm{A})}+\frac{\sqrt{3}}{2}\left(e^{\infty}-e_{\mathrm{L}}\right)\right)\right]^{\frac{1}{n}}
$$

\section{A.3. Hexagonal lattice and diamond lattice}

The hexagonal lattice is bending-dominated in uniaxial tension. In contrast, the diamond lattice is strongly anisotropic: it is stretching-dominated when loaded along the strut directions, but is otherwise bending-dominated. For tensile loading along the $x_{2}$-direction of Figs. 1(c)-(d), both lattices deform in essentially the same manner: the predominant mode of deformation in regimes I and II is bending of the inclined struts A and B. These struts behave as built-in beams of length $\ell_{0} / 2$ and width $b$ subjected to a transverse tip load $P$ at free end, see for example Gibson and Ashby (1997). Consequently, the macroscopic response of the 
lattice can be estimated from the end deflection of a single half-strut A (or B). Simple beam theory gives the tip deflection $\delta$ for linear-elastic regime I as (Timoshenko and Gere 1961),

$$
\delta=\frac{P}{2 b E_{S}}\left(\frac{\ell_{0}}{t_{0}}\right)^{3}
$$

and the maximum strain $\varepsilon^{(A)}$ (which occurs at the built-in end of the beam) is

$$
\varepsilon^{(\mathrm{A})}=\frac{3 P \ell_{0}}{E_{S} b t_{0}^{2}}
$$

Now, by the method of sections, the macroscopic nominal stress $s^{\infty}$ for each lattice topology is related to $P$ by

$$
s^{\infty}=\frac{P}{\ell_{0} b \cos ^{2} \alpha}
$$

where $\alpha$ is the inclination of strut $\mathrm{A}$ with the $x_{1}$ axis: $\alpha=\pi / 6$ for the regular hexagonal lattice, and $\alpha=\pi / 4$ for the diamond lattice. Similarly, the nominal strain $e^{\infty}$ is related to the mid-span defection $\delta$ of strut A as

$$
e^{\infty}=\frac{2 \delta \cos \alpha}{\ell_{0}\left(c_{0}+\sin \alpha\right)}
$$

where the geometric parameter $c_{0}=1$ for the hexagonal lattice and $c_{0}=0$ for the diamond lattice.

Combining relations (A.30) through (A.33) gives the macroscopic response in regime I as

$$
s^{\infty}=c_{1} \bar{\rho}^{3} E_{\mathrm{S}} e^{\infty}
$$

where $c_{1}=3 / 2$ and $\bar{\rho}=2 t_{0} / \sqrt{3} \ell_{0}$ for the hexagonal lattice and $c_{1}=1 / 4$ and $\bar{\rho}=$ $2 t_{0} / \ell_{0}$ for the diamond lattice. The maximum bending strain in struts $\mathrm{A}$ and $\mathrm{B}$, upon combining equations (A.30), (A.31), and (A.33) reads

$$
\varepsilon^{(\mathrm{A})}=\varepsilon^{(\mathrm{B})}=d_{1} \bar{\rho} e^{\infty}
$$

where $d_{1}=9 / 2$ for the hexagonal lattice and $d_{1}=3 / 2$ for the diamond lattice.

In the initial part of regime II where the cell wall bending strain $\varepsilon^{(\mathrm{A})}$ is small and comparable to the macroscopic strain $e^{\infty}$, it is adequate to treat the cell wall response as a Ramberg-Osgood power-law relation i.e., omitting the $\exp (\varepsilon)$ term of Eq. (A.1). The corresponding corresponding relations for Eq. (A.30) and Eq. (A.31) in regime II then become

$$
\frac{\delta}{\varepsilon_{0 S} t_{0}}=\left(\frac{2 n+1}{n}\right)^{n} \frac{1}{2(n+2)}\left(\frac{P}{\sigma_{0 S} b t_{0}}\right)^{n}\left(\frac{\ell_{0}}{t_{0}}\right)^{n+2}
$$

and 


$$
\frac{\varepsilon^{(\mathrm{A})}}{\varepsilon_{0 \mathrm{~S}}}=\left(\frac{2 n+1}{n}\right)^{n}\left(\frac{P}{\sigma_{0 S} b t_{0}}\right)^{n}\left(\frac{\ell_{0}}{t_{0}}\right)^{n}
$$

Now upon making use of Eq. (A.36), Eqs. (A.32) and (A.33) give the macroscopic response in regime II as

$$
\frac{s^{\infty}}{\sigma_{0 S}}=c_{2} \frac{n}{2 n+1} \bar{\rho}^{\frac{2 n+1}{n}}\left[\frac{n+2}{2} \frac{e^{\infty}}{\varepsilon_{0 S}}\right]^{\frac{1}{n}}
$$

where $c_{2}=3^{1 / n}$ for the hexagonal lattice and $c_{2}=1 / 2$ for the diamond lattice. Likewise, the maximum bending strain in struts $\mathrm{A}$ and $\mathrm{B}$ is obtained by dividing Eq. (A.37) by Eq. (A.36), and then making use of Eq. (A.33) to give

$$
\varepsilon^{(\mathrm{A})}=\varepsilon^{(\mathrm{B})}=d_{2}(n+2) \bar{\rho} e^{\infty}
$$

where $d_{2}=3 / 2$ for the hexagonal lattice and $d_{2}=1 / 2$ for the diamond lattice.

At large values of $e^{\infty}$, strut $\mathrm{B}$ rotates to align with the loading axis $x_{2}$; this marks the beginning of regime III. The hexagonal lattice locks-up at a nominal lock-up strain $e_{\mathrm{L}}=1 / 3$ and the diamond lattice locks up at $e_{\mathrm{L}}=\sqrt{2}-1$. In regime III $\left(e^{\infty}>e_{\mathrm{L}}\right)$, all cell walls undergo stretching. Write $\left(\sigma^{(\mathrm{A})}, \varepsilon^{(\mathrm{A})}\right)$ and $\left(\sigma^{(\mathrm{C})}, \varepsilon^{(\mathrm{C})}\right)$ as the true axial stress and strain in strut A (and B) and strut C, respectively. Note that struts A and B of the hexagonal lattice carry only half the tensile load as carried by strut C. Consequently, the axial strain in struts A and B is negligible, and strut $\mathrm{C}$ stretches according to

$$
\varepsilon^{(\mathrm{C})}=\ln \frac{\ell^{(\mathrm{C})}}{\ell_{0}}=\ln \left(1+\frac{3}{2}\left(e^{\infty}-e_{\mathrm{L}}\right)\right)
$$

The corresponding true stress in the strut is given by

$$
\sigma^{(\mathrm{C})}=\frac{2}{\bar{\rho}}\left(1+\frac{3}{2}\left(e^{\infty}-e_{\mathrm{L}}\right)\right) s^{\infty}
$$

Now substitute relations (A.40) and (A.41) into Eq. (A.1) to give the nominal stress versus strain response of the hexagonal lattice in regime III as

$$
\frac{s^{\infty}}{\sigma_{0 \mathrm{~S}}}=0.5 \bar{\rho}\left[\frac{1}{\varepsilon_{0 \mathrm{~S}}} \ln \left(1+\frac{3}{2}\left(e^{\infty}-e_{\mathrm{L}}\right)\right)\right]^{\frac{1}{n}}
$$

The struts $\mathrm{A}$ and $\mathrm{B}$ of the diamond lattice stretch by

$$
\varepsilon^{(\mathrm{A})}=\varepsilon^{(\mathrm{B})}=\ln \frac{\ell^{(\mathrm{A})}}{\ell_{0}}=\ln \left(1+\frac{1}{\sqrt{2}}\left(e^{\infty}-e_{\mathrm{L}}\right)\right)
$$

Joint equilibrium in the locked-up state of the unit cell gives

$$
\sigma^{(\mathrm{A})}=\frac{\sqrt{2}}{\bar{\rho}}\left(1+\frac{1}{\sqrt{2}}\left(e^{\infty}-e_{\mathrm{L}}\right)\right) s^{\infty}
$$

Now substitute relations (A.43) and (A.44) into Eq. (A.1) to obtain the macroscopic response 
of the diamond lattice in regime III as

$$
\frac{s^{\infty}}{\sigma_{0 \mathrm{~S}}}=\frac{\bar{\rho}}{\sqrt{2}}\left[\frac{1}{\varepsilon_{0 \mathrm{~S}}} \ln \left(1+\frac{1}{\sqrt{2}}\left(e^{\infty}-e_{\mathrm{L}}\right)\right)\right]^{\frac{1}{n}}
$$

\section{REFERENCES}

Anderson, T.L., 1995. Fracture Mechanics: Fundamentals and Applications, Aerospace Engineering. CRC Press.

Calladine, C.R., English, R.W., 1984. Strain-rate and inertia effects in the collapse of two types of energy-absorbing structure. Int. J. Mech. Sci. 26, 689-701.

Chen, C., Fleck, N.A., Lu, T.J., 2001. The mode I crack growth resistance of metallic foams 49, 231-259.

Chen, C., Lu, T.J., Fleck, N.A., 1999. Effect of imperfections on the yielding of twodimensional foams. J. Mech. Phys. Solids 47, 2235-2272.

Côté, F., Deshpande, V.S., Fleck, N.A., Evans, A.G., 2006. The shear response of metallic square honeycombs. J. Mech. Mater. Struct. 1, 1281-1299.

Côté, F., Deshpande, V.S., Fleck, N.A., Evans, A.G., 2004. The out-of-plane compressive behavior of metallic honeycombs. Mater. Sci. Eng. A 380, 272-280.

de Kruijf, N.E., Peerlings, R.H.J., Geers, M.G.D., 2009. An analysis of sheet necking under combined stretching and bending. Int. J. Mater. Form. 2, 845-848.

Deshpande, V.S., Ashby, M.F., Fleck, N.A., 2001. Foam topology bending versus stretching dominated architectures 49, 1035-1040.

Engesser, F., 1889. Ueber die knickfestigkeit gerader st“abe. Zeitschrift f“ur Archit. und Ingenieurwes. 35, 455-462.

Fleck, N.A., Deshpande, V.S., Ashby, M.F., 2010. Micro-Architectured Materials: Past, Present and Future. Proc. R. Soc. A Math. Phys. Eng. Sci. 466, 2495-2516.

Fleck, N.A., Qiu, X., 2007. The damage tolerance of elastic-brittle, two-dimensional isotropic lattices. J. Mech. Phys. Solids 55, 562-588.

Gibson, J.W., Ashby, M.F., 1997. Cellular Solids: Structures and Properties. Cambridge University Press.

Grediac, M., 1993. A finite element study of the transverse shear in honeycomb cores. Int. J. Solids Struct. 30, 1777-1788.

Hidalgo, R.C., Hermann, H.J., Parteli, E.J.R., Kun, F., 2004. Varenna Proceedings Course CLV, in: Mallamace, F.; Stanley, H.E. (Ed.), The Physics of Complex Systems. IOS 
Press, Amsterdam, pp. 153-171.

Hutchinson, R.G., Fleck, N.A., 2006. The structural performance of the periodic truss. J. Mech. Phys. Solids 54, 756-782.

Hutchinson, R.G., Wicks, N., Evans, A.G., Fleck, N.A., Hutchinson, J.W., 2003. Kagome plate structures for actuation. Int. J. Solids Struct. 40, 6969-6980.

Hyun, S., Torquato, S., 2002. Optimal and manufacturable two-dimensional, Kagome-like cellular solids. J. Mater. Res. 17, 137-144.

Katbab, A.A., Nazockdast, H., Bazgir, S., 2000. Carbon black-reinforced dynamically cured EPDM/PP thermoplastic elastomers. I. Morphology, rheology, and dynamic mechanical properties. J. Appl. Polym. Sci. 75, 1127-1137.

Liu, A., 1996. Summary of stress Intensity Factors. ASM Int.

Ma, Q., Cheng, H., Jang, K.I., Luan, H., Hwang, K.C., Rogers, J.A., Huang, Y., Zhang, Y., 2016. A nonlinear mechanics model of bio-inspired hierarchical lattice materials consisting of horseshoe microstructures. J. Mech. Phys. Solids 90, 179-202.

Mangipudi, K.R., Onck, P.R., 2012. Tensile failure of two-dimensional quasi-brittle foams, in: International Journal of Solids and Structures. pp. 2823-2829.

Mangipudi, K.R., Onck, P.R., 2011. Notch Sensitivity of Ductile Metallic Foams: A Computational Study. Acta Mater. 59, 7356-7367.

Olurin, O.B., Fleck, N.A., Ashby, M.F., 2000. Deformation and fracture of aluminium foams. Mater. Sci. Eng. A 291, 136-146.

Onck, P.R., Van Merkerk, R., De Hosson, J.T.M., Schmidt, I., 2004. Fracture of Metal Foams: In-Situ Testing and Numerical Modeling. Adv. Eng. Mater. 6, 429-431.

Papka, S.D., Kyriakides, S., 1994. In-plane compressive response and crushing of honeycomb. J. Mech. Phys. Solids 42, 1499-1532.

Poon, B.C., Dias, P., Ansems, P., Chum, S.P., Hiltner, A., Baer, E., 2007. Structure and deformation of an elastomeric propylene-ethylene copolymer. J. Appl. Polym. Sci. 104, 489-499.

Quintana Alonso, I., Fleck, N.A., 2007. Damage Tolerance of an Elastic-Brittle DiamondCelled Honeycomb. Scr. Mater. 56, 693-696.

Quintana Alonso, I., Mai, S.P., Fleck, N.A., Oakes, D.C.H., Twigg, M. V., 2010. The fracture toughness of a cordierite square lattice. Acta Mater. 58, 201-207.

Romijn, N.E.R., Fleck, N.A., 2007. The fracture toughness of planar lattices: Imperfection sensitivity. J. Mech. Phys. Solids 55, 2538-2564.

Sih, G.C., Paris, P.C., Irwin, G.R., 1965. On cracks in rectilinearly anisotropic bodies. Int. J. 
Fract. Mech. 1, 189-203.

Symons, D.D., Fleck, N.A., 2008. The imperfection sensitivity of isotropic two-dimensional elastic lattices. J. Appl. Mech. 75, 51011.

Tankasala, H.C., Deshpande, V.S., Fleck, N.A., 2015. 2013 Koiter Medal Paper: Crack-tip fields and toughness of two-dimensional elastoplastic lattices. J. Appl. Mech. 82, 910041-10.

Timoshenko, S. P. and Gere, J.M., 1961. Theory of Elastic Stability, 2nd ed. McGraw-Hill, New York.

Tse, M.F., Jiang, P., 2013. Thermoplastic Polyolefin Blends. US Patent 8,410,217 B2.

Wang, A.-J., McDowell, D.L., 2004. In-plane stiffness and yield strength of periodic metal honeycombs. J. Eng. Mater. Technol. 126, 137.

Yang, M.Y., Huang, J.S., 2005. Elastic buckling of regular hexagonal honeycombs with plateau borders under biaxial compression. Compos. Struct. 71, 229-237.

Zhu, H., Hobdell, J.R., Windle, A.H., 2001. Effects of cell irregularity on the elastic properties of 2D Voronoi honeycombs. J. Mech. Phys. Solids 49, 857-870. 


\section{LIST OF TABLES}

Table 1. Coefficients for relative density $\bar{\rho}$, elastic modulus $E$, ultimate tensile strength $s_{\mathrm{f}}^{\infty}$ (for elastic-brittle case), and plastic collapse strength $s_{\mathrm{Y}}$ (for perfectly plastic case).

Table 2. Primary mode of deformation within each strut of the elastoplastic lattice, for uniaxial tension (in the $x_{2}$-direction).

Table 3. Coefficients for lattice ductility $e_{\mathrm{f}}^{\infty}$ and ultimate tensile strength $s_{\mathrm{f}}^{\infty}$ in regime II.

Table 4. Coefficients for transition flaw size $a_{\mathrm{Ty}} / \ell_{0}$ of the elastoplastic lattice according to LTS criterion. 


\section{LIST OF FIGURES}

Fig. 1. Lattice topologies and the corresponding unit cells employed for the FE analyses, for (a) triangular lattice; (b) Kagome lattice; (c) hexagonal lattice; and (d) diamond lattice.

Fig. 2. Uniaxial true stress versus true strain response of the cell wall material as given by Eq. (6) for selected values of strain hardening exponent $n$.

Fig. 3. Macroscopic nominal stress versus strain response of triangular lattice under uniaxial tension, for the case $\bar{\rho}=0.1, \varepsilon_{0 \mathrm{~S}}=0.001$ and $n=10$.

Fig. 4. Uniaxial tensile response of the Kagome lattice. (a) nominal stress versus nominal strain; and (b) cell wall true strain versus macroscopic nominal strain, for the case $\bar{\rho}=0.1$, $\varepsilon_{0 \mathrm{~S}}=0.001$ and $n=10$.

Fig. 5. Full response of a single strut subjected to axial compressive load. (a) end conditions on the strut; and load versus shortening response for selected values of (b) $t_{0} / \ell_{0}$ and (c) $n$; (d) coefficient of the Maxwell estimate for the average load in single strut $(Q)$ and for the constant tensile stress in regime IIb of the Kagome lattice $(R)$.

Fig. 6. Modes of plastic instability in regime II of the Kagome lattice. (a) macroscopic strain at the onset of rupture (in struts A and B) and buckling (of strut C); and (b) a map showing the contours of macroscopic strain at the onset of instability with axes $(1 / n, \bar{\rho})$.

Fig. 7. Uniaxial tensile response of the hexagonal lattice. (a) nominal stress versus nominal strain; and (b) cell wall true strain versus macroscopic nominal strain, for the case $\bar{\rho}=0.1$, $\varepsilon_{0 \mathrm{~S}}=0.001$ and $n=10$.

Fig. 8. Macroscopic nominal stress versus strain response of the diamond lattice under uniaxial tension, for the case $\bar{\rho}=0.1, \varepsilon_{0 \mathrm{~S}}=0.001$ and $n=10$. 
Fig. 9. A summary of the tensile response of the four lattices. (a) nominal stress versus nominal strain response; macroscopic ductility versus cell wall failure strain according to (b) LTS criterion and (c) ATS criterion; and (d) ultimate tensile strength versus cell wall failure strain according to LTS and ATS criteria, for the case $\bar{\rho}=0.1, \varepsilon_{0 S}=0.001$ and $n=10$.

Fig. 10. Random lattice topologies with $R / \ell_{0}=0.5$.

Fig. 11. A comparison of the macroscopic ductility of the four random lattices as a function of the degree of imperfection $R / \ell_{0}$, according to (a) LTS criterion and (b) ATS criterion. The error bars in each case correspond to the standard error for a sample of 10 realisations.

Fig 12. A comparison of the deformed meshes (and contours of axial stress) at the instant when the ATS criterion is met in (a) random hexagonal lattice, and (b) random diamond lattice. For both lattices, $R / \ell_{0}=0.5$.

Fig. 13. Centre-cracked plate made from an elastoplastic lattice subjected to uniaxial tension. (a) Geometry and loading; crack morphologies for (b) triangular lattice; and (c) hexagonal lattice. The observed failure site in both the lattices is indicated by $\mathrm{x}$ in parts (b) and (c).

Fig. 14. Notch tensile strength of the elastoplastic lattices as a function of crack length for the choice $\bar{\rho}=0.05$ and $n=10$. (a) triangular lattice with LTS criterion; (b) hexagonal lattice with LTS criterion; (c) triangular lattice with ATS criterion; and (d) hexagonal lattice with ATS criterion. The Dugdale prediction Eq. (21) is shown by dashed lines.

Fig. 15. Notch sensitivity of elastic-brittle lattices: (a) triangular lattice; and (b) hexagonal lattice. The Dugdale prediction, Eq. (24), is shown by dashed lines. 
Table 1. Coefficients for relative density $\bar{\rho}$, elastic modulus $E$, ultimate tensile strength $s_{\mathrm{f}}^{\infty}$ (for elastic-brittle case), and plastic collapse strength $s_{\mathrm{Y}}$ (for perfectly plastic case).

\begin{tabular}{|l|l|l|l|l|l|l|}
\hline Lattice & $A$ & $B$ & $b$ & $C_{1}$ & $C_{2}$ & $c$ \\
\hline Triangular & $2 \sqrt{3}$ & $1 / 3$ & 1 & $1 / 3$ & $1 / 3$ & 1 \\
\hline Kagome & $\sqrt{3}$ & $1 / 3$ & 1 & $1 / 2$ & $1 / 2$ & 1 \\
\hline Hexagonal & $2 / \sqrt{3}$ & $3 / 2$ & 3 & $1 / 3$ & $1 / 2$ & 2 \\
\hline Diamond & 2 & $1 / 4$ & 3 & $1 / 6$ & $1 / 4$ & 2 \\
\hline
\end{tabular}

Table 2. Primary mode of deformation within each strut of the elastoplastic lattice, for uniaxial tension (in the $x_{2}$-direction).

\begin{tabular}{|c|c|c|c|c|c|}
\hline \multirow{2}{*}{ Lattice } & \multirow{2}{*}{ Strut } & \multicolumn{3}{|c|}{ Regime } & \multirow[b]{2}{*}{ III } \\
\hline & & $\mathrm{I}$ & IIa & $\mathrm{IIb}$ & \\
\hline \multirow[t]{2}{*}{ Triangular } & A, B & Elastic bending & $\begin{array}{l}\text { Elastic bending } \\
\text { and then plastic } \\
\text { bending }\end{array}$ & \multirow[t]{2}{*}{-} & $\begin{array}{l}\text { Plastic } \\
\text { stretching }\end{array}$ \\
\hline & $\mathrm{C}$ & Elastic stretching & $\begin{array}{l}\text { Plastic } \\
\text { stretching }\end{array}$ & & $\begin{array}{l}\text { Plastic } \\
\text { stretching }\end{array}$ \\
\hline \multirow[b]{2}{*}{ Kagome } & A, B & Elastic stretching & $\begin{array}{l}\text { Plastic } \\
\text { stretching }\end{array}$ & $\begin{array}{l}\text { Rotation } \\
\text { without stretch }\end{array}$ & $\begin{array}{l}\text { Plastic } \\
\text { stretching }\end{array}$ \\
\hline & $\mathrm{C}$ & Elastic shortening & $\begin{array}{l}\text { Elastic } \\
\text { shortening then } \\
\text { plastic } \\
\text { shortening }\end{array}$ & $\begin{array}{l}\text { Plastic } \\
\text { buckling }\end{array}$ & $\begin{array}{l}\text { Plastic } \\
\text { buckling }\end{array}$ \\
\hline \multirow{2}{*}{ Hexagonal } & A, B & Elastic bending & Plastic bending & \multirow{2}{*}{-} & $\begin{array}{l}\text { Plastic } \\
\text { stretching }\end{array}$ \\
\hline & $\mathrm{C}$ & Elastic stretching & $\begin{array}{l}\text { Elastic } \\
\text { stretching }\end{array}$ & & $\begin{array}{l}\text { Plastic } \\
\text { stretching }\end{array}$ \\
\hline
\end{tabular}


Table 3. Coefficients for lattice ductility $e_{\mathrm{f}}^{\infty}$ and ultimate tensile strength $s_{\mathrm{f}}^{\infty}$ in regime II.

\begin{tabular}{|l|l|l|l|l|}
\hline Lattice & $D$ & $d$ & $C$ & $c$ \\
\hline Triangular & 1 & 0 & $1 / 3$ & 1 \\
\hline Kagome & $\frac{1}{3} \frac{2^{n+2}+1}{2^{n}}$ & 0 & $1 / 2$ & 1 \\
\hline Hexagonal & $\frac{2}{3(n+2)}$ & -1 & $\frac{n}{2 n+1}$ & 2 \\
\hline Diamond & $\frac{2}{n+2}$ & -1 & $\frac{n}{2(2 n+1)}$ & 2 \\
\hline
\end{tabular}

Table 4. Coefficients for transition flaw size $a_{\mathrm{Ty}} / \ell_{0}$ of the elastoplastic lattice according to LTS criterion.

\begin{tabular}{|l|l|l|l|l|}
\hline \multirow{2}{*}{ Lattice } & \multicolumn{3}{|c|}{$H$} & \multirow{2}{*}{$h$} \\
\cline { 2 - 4 } & $n=3$ & $n=10$ & $n=\infty$ & \\
\hline Triangular & 0.76 & 0.67 & 0.59 & 0 \\
\hline Kagome & 0.056 & 0.05 & 0.02 & -1 \\
\hline Hexagonal & 0.74 & 0.34 & 0.073 & 0 \\
\hline Diamond & 0.22 & 0.18 & 0.1 & -2 \\
\hline
\end{tabular}


a

Triangular lattice

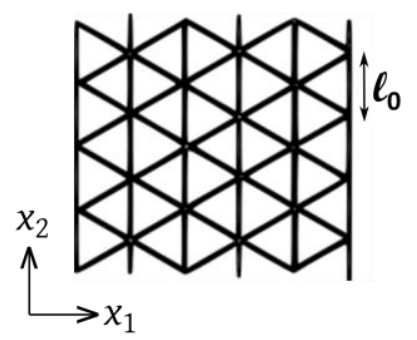

C

Hexagonal lattice

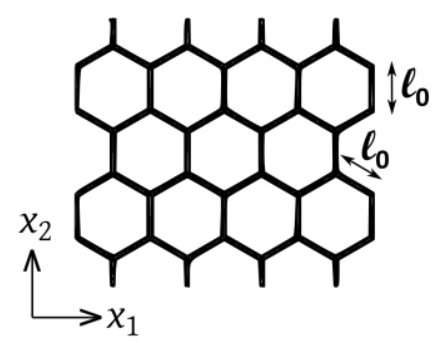

Unit cell

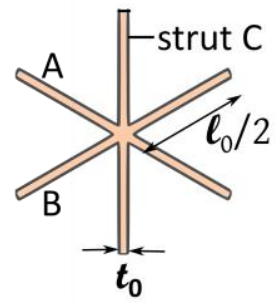

Unit cell

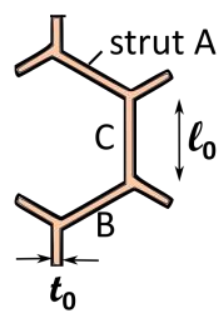

b

Kagome lattice

Unit cell
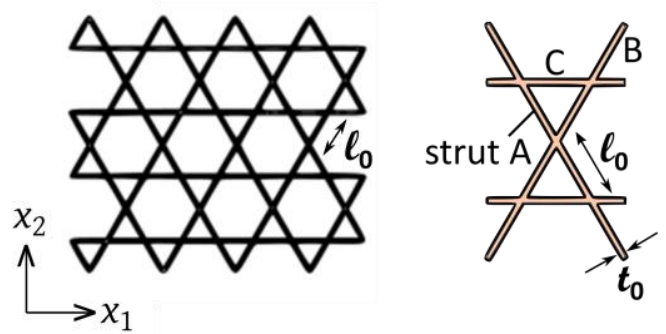

d

Diamond lattice

Unit cell

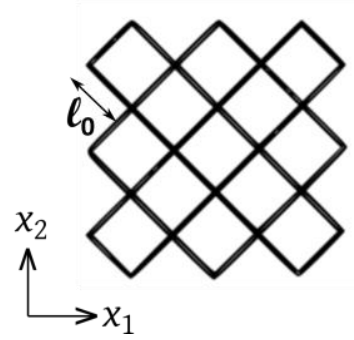

Fig. 1. Lattice topologies and the corresponding unit cells employed for the FE analyses, for (a) triangular lattice; (b) Kagome lattice; (c) hexagonal lattice; and (d) diamond lattice.

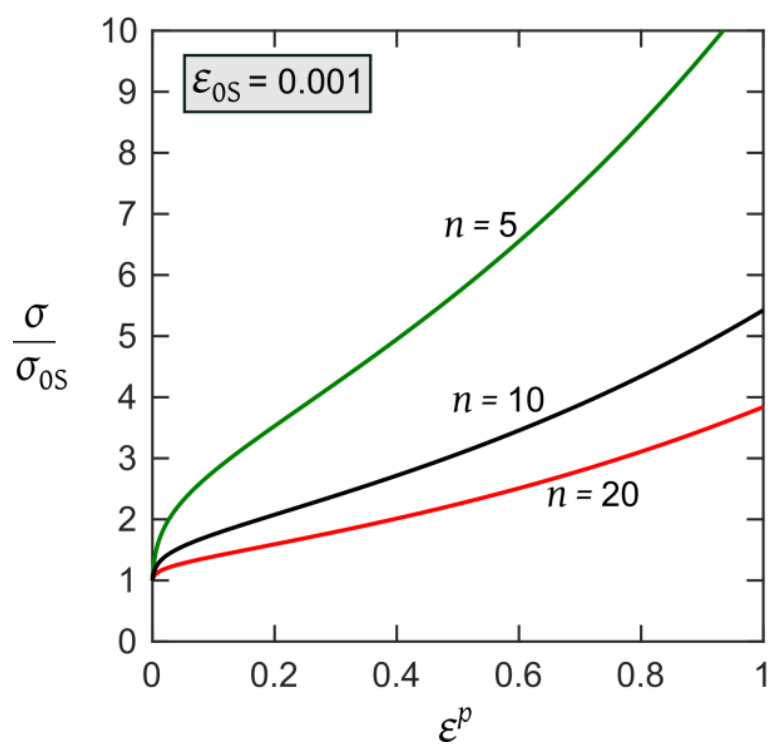

Fig. 2. Uniaxial true stress versus true strain response of the cell wall material as given by Eq. (6) for selected values of strain hardening exponent $n$. 


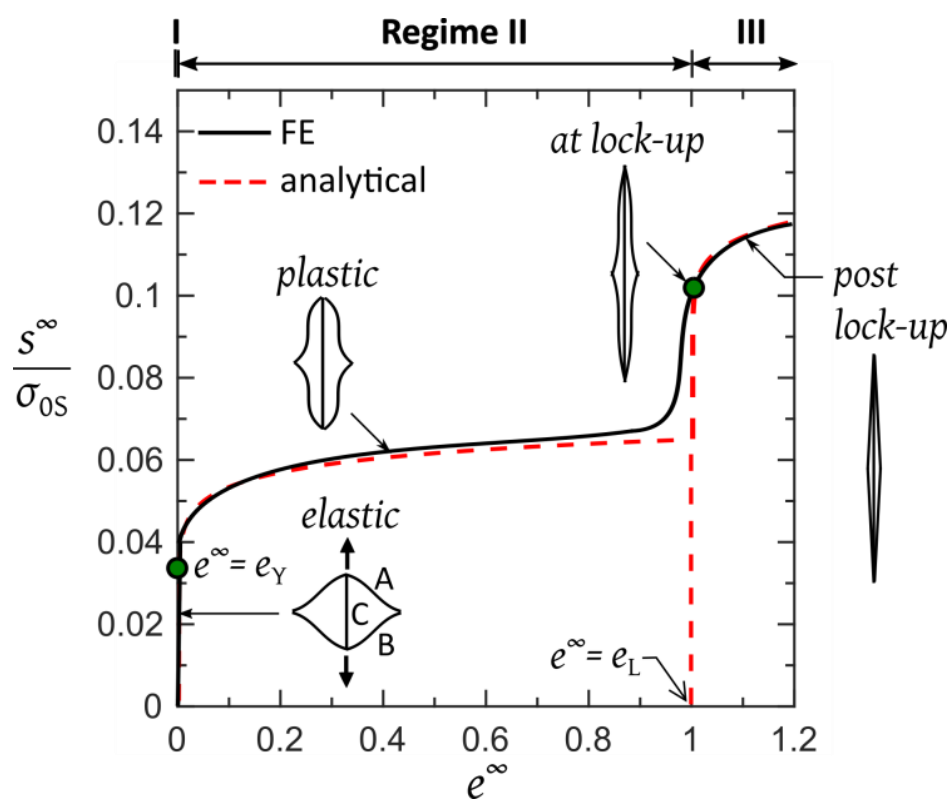

Fig. 3. Macroscopic nominal stress versus strain response of triangular lattice under uniaxial tension, for the case $\bar{\rho}=0.1, \varepsilon_{0 \mathrm{~S}}=0.001$ and $n=10$.

a

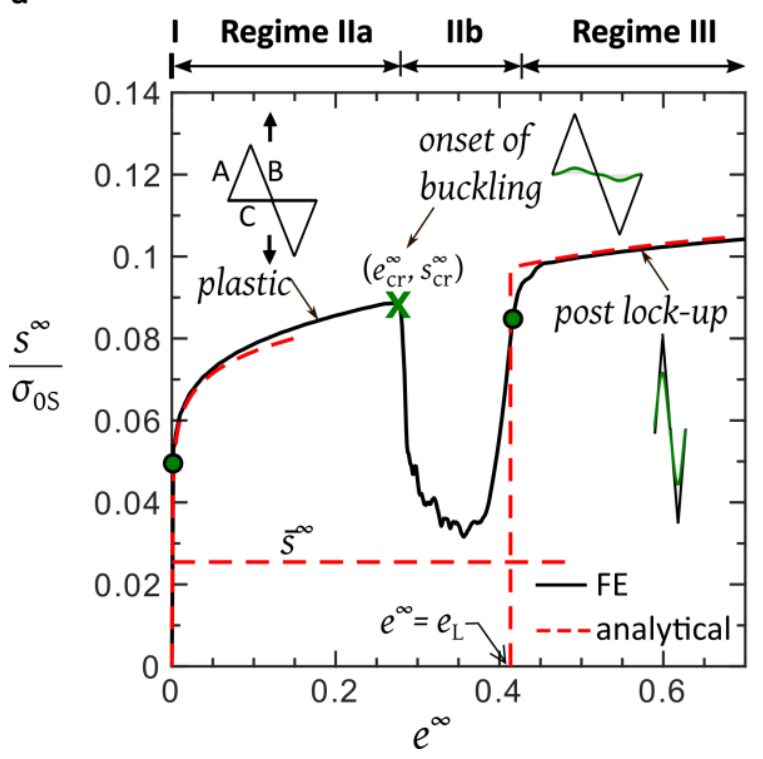

b

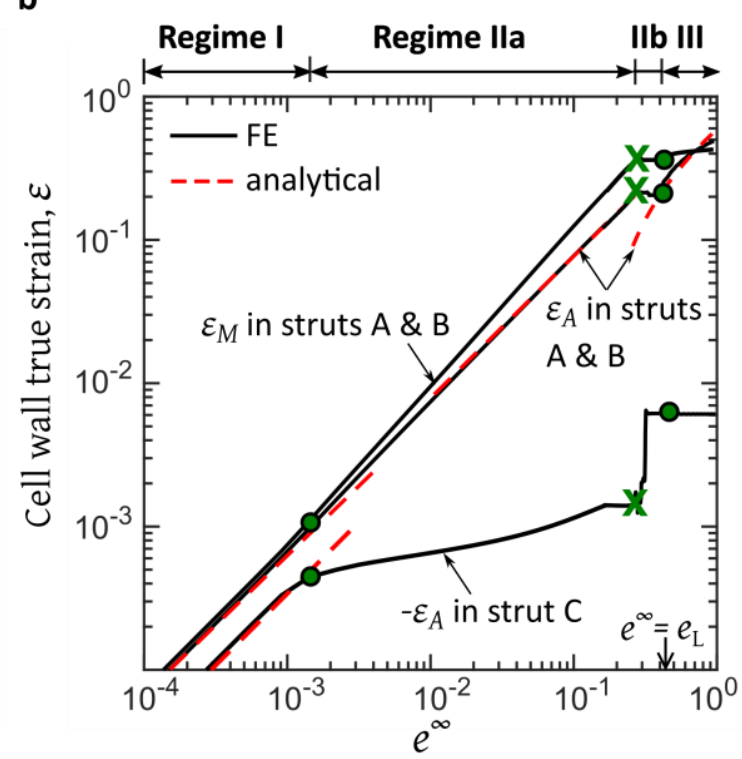

Fig. 4. Uniaxial tensile response of the Kagome lattice. (a) nominal stress versus nominal strain; and (b) cell wall true strain versus macroscopic nominal strain, for the case $\bar{\rho}=0.1$, $\varepsilon_{0 \mathrm{~S}}=0.001$ and $n=10$. 
b

a
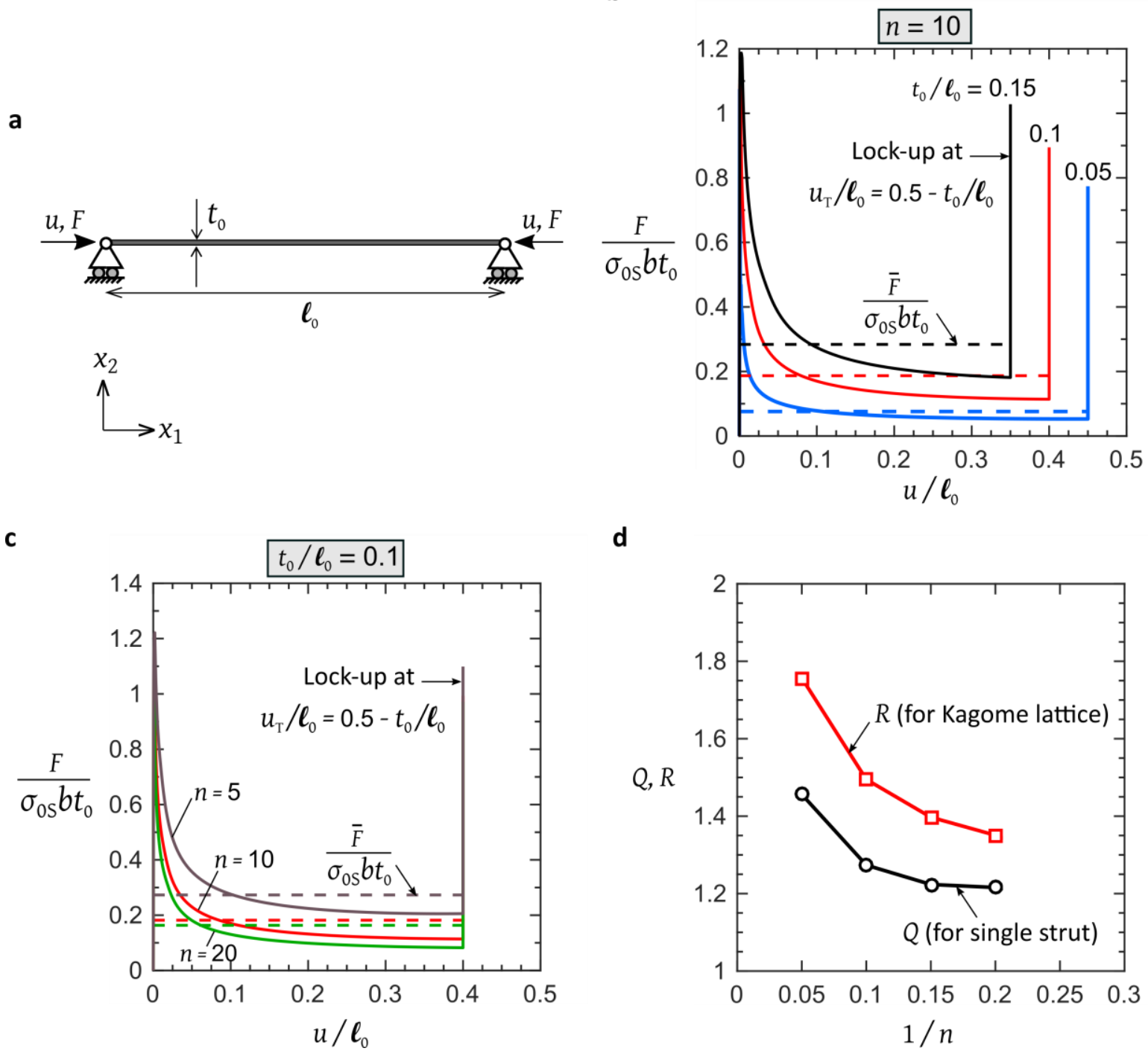

d

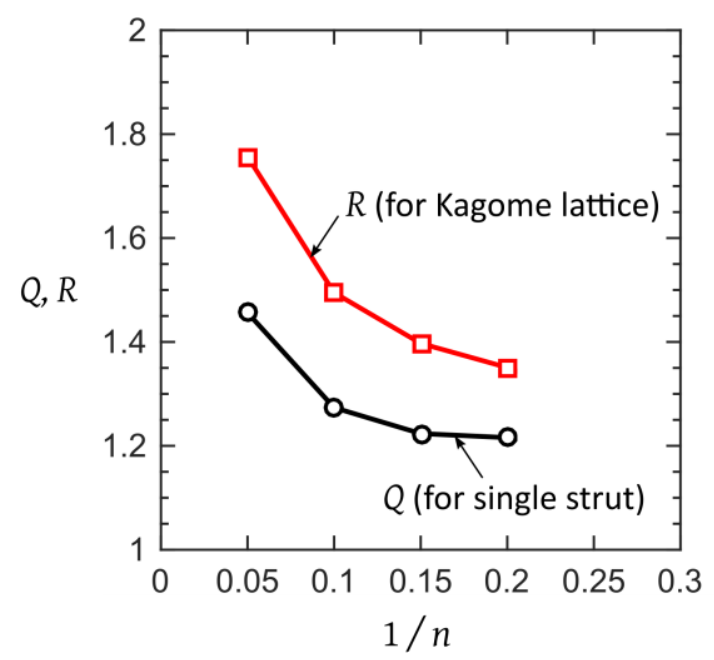

Fig. 5. Full response of a single strut subjected to axial compressive load. (a) end conditions on the strut; and load versus shortening response for selected values of (b) $t_{0} / \ell_{0}$ and (c) $n$; (d) coefficient of the Maxwell estimate for the average load in single strut $(Q)$ and for the constant tensile stress in regime IIb of the Kagome lattice $(R)$. 
a

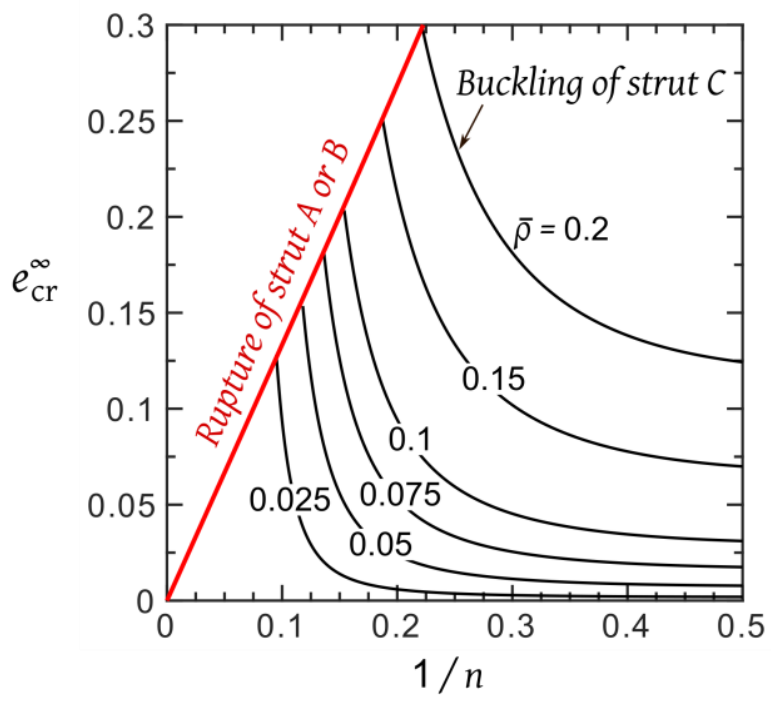

b

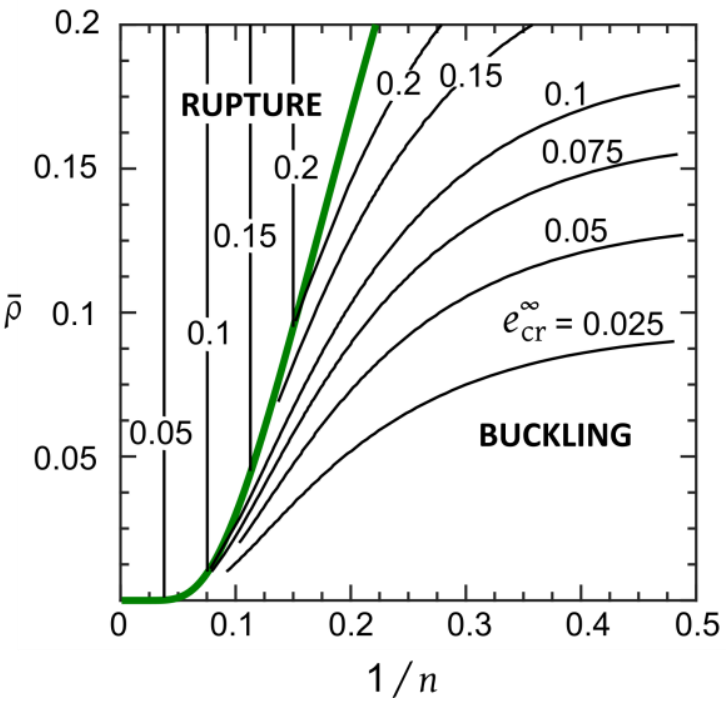

Fig. 6. Modes of plastic instability in regime II of the Kagome lattice. (a) macroscopic strain at the onset of rupture (in strut A or B) and buckling (of strut C); and (b) a map showing the contours of macroscopic strain at the onset of instability with axes $(1 / n, \bar{\rho})$.

a

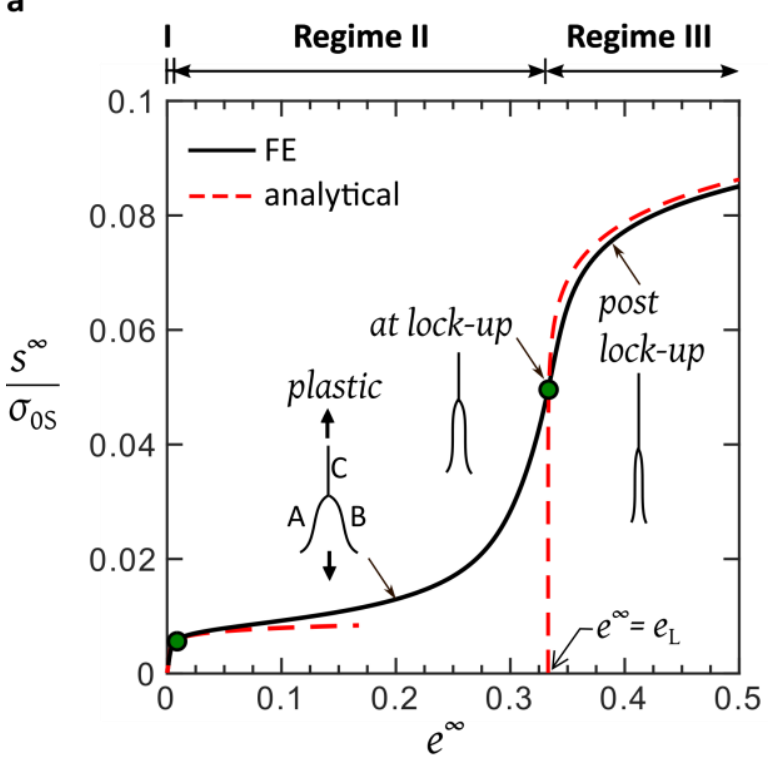

b

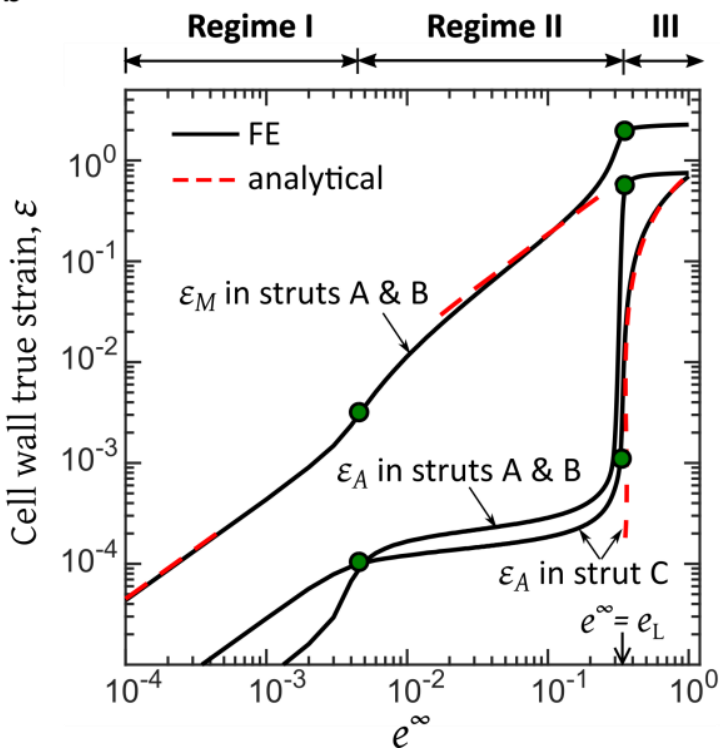

Fig. 7. Uniaxial tensile response of the hexagonal lattice: (a) nominal stress versus nominal strain; and (b) cell wall true strain versus macroscopic nominal strain, for the case $\bar{\rho}=0.1$, $\varepsilon_{0 S}=0.001$ and $n=10$. 


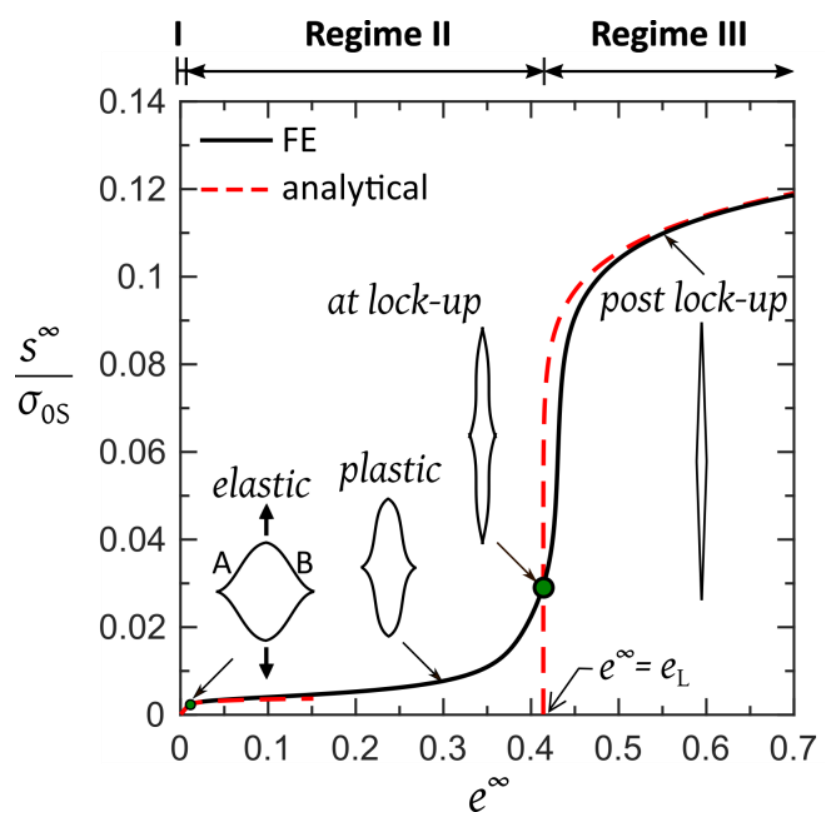

Fig. 8. Macroscopic nominal stress versus strain response of the diamond lattice under uniaxial tension, for the case $\bar{\rho}=0.1, \varepsilon_{0 \mathrm{~S}}=0.001$ and $n=10$. 


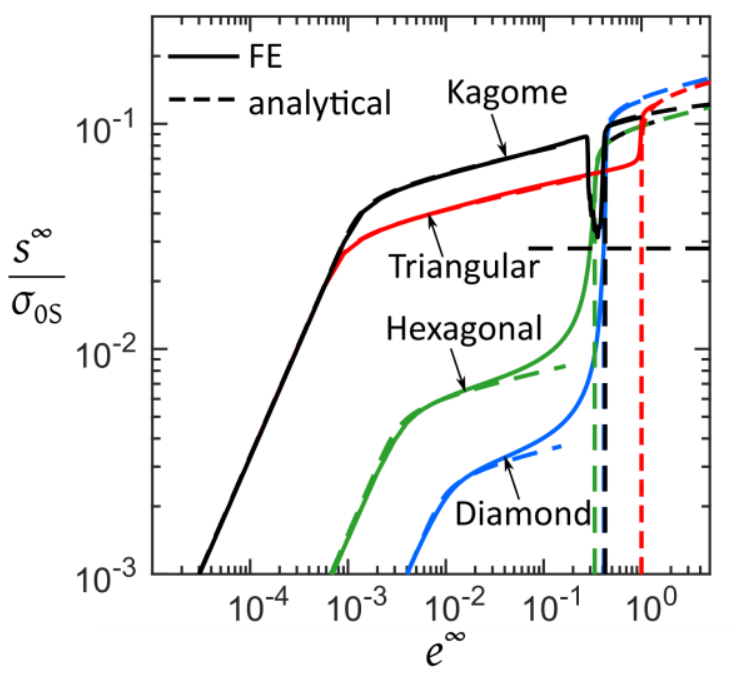

C

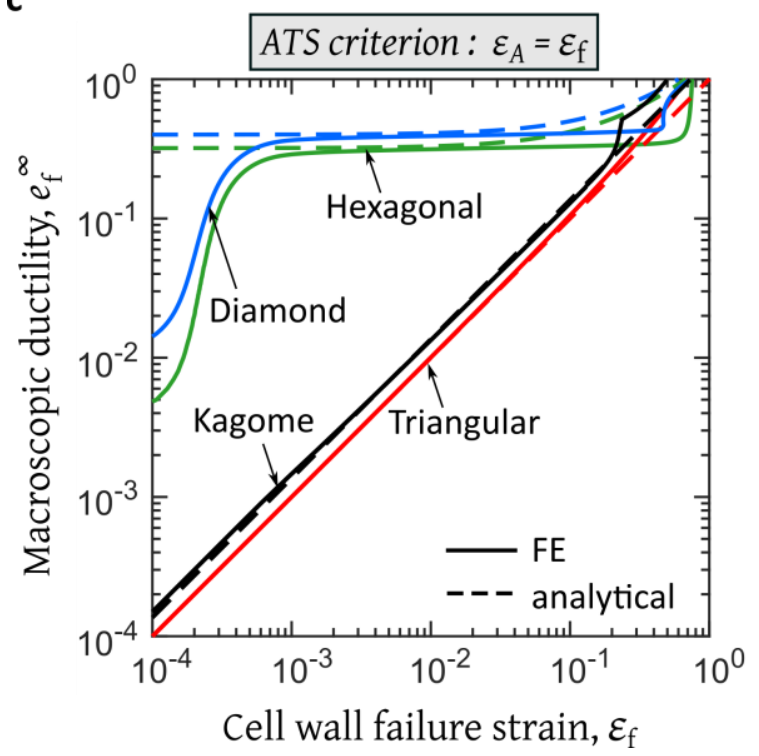

b

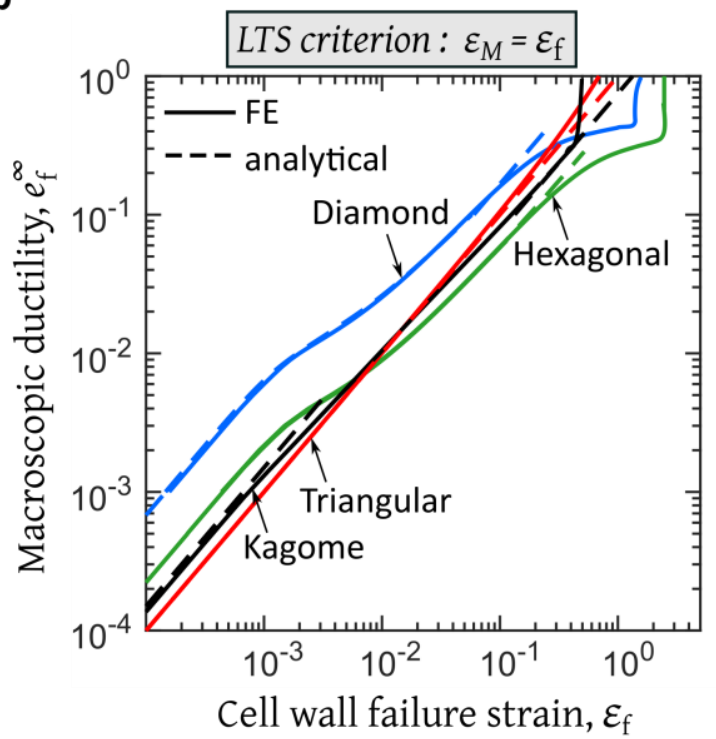

d

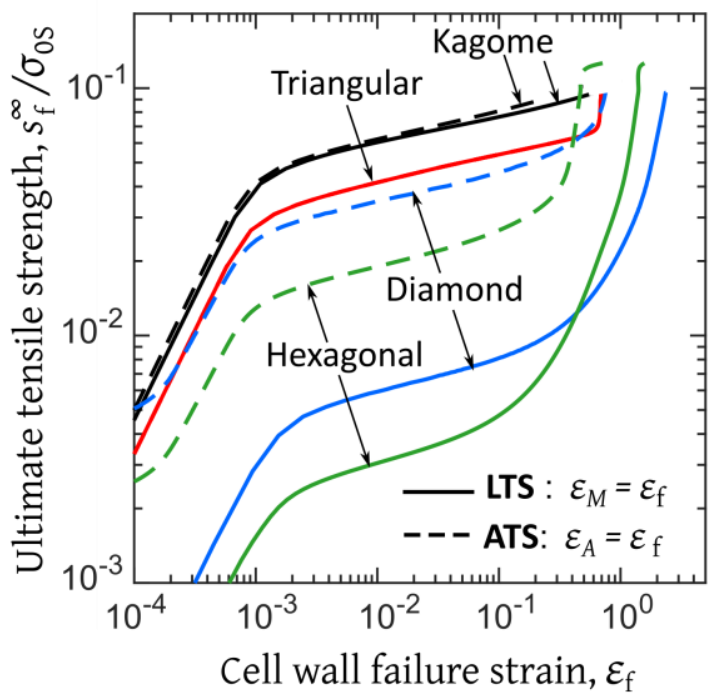

Fig. 9. A summary of the tensile response of the four lattices. (a) nominal stress versus nominal strain response; macroscopic ductility versus cell wall failure strain according to (b) LTS criterion and (c) ATS criterion; and (d) ultimate tensile strength versus cell wall failure strain according to LTS and ATS criteria, for the case $\bar{\rho}=0.1, \varepsilon_{0 \mathrm{~S}}=0.001$ and $n=10$. 
a

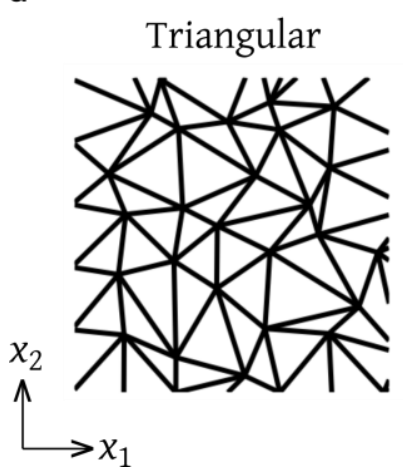

b

Kagome

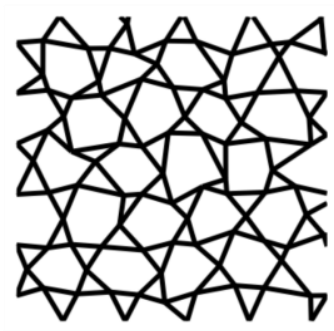

Hexagonal

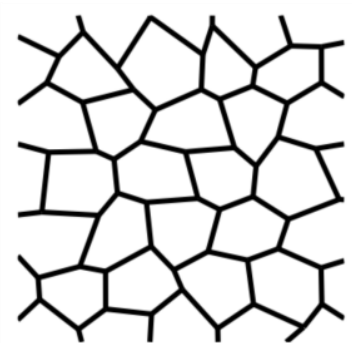

d

Diamond

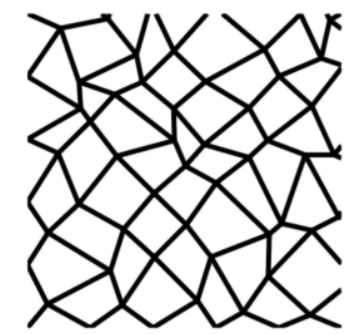

Fig. 10. Random lattice topologies with $R / \ell_{0}=0.5$.

a

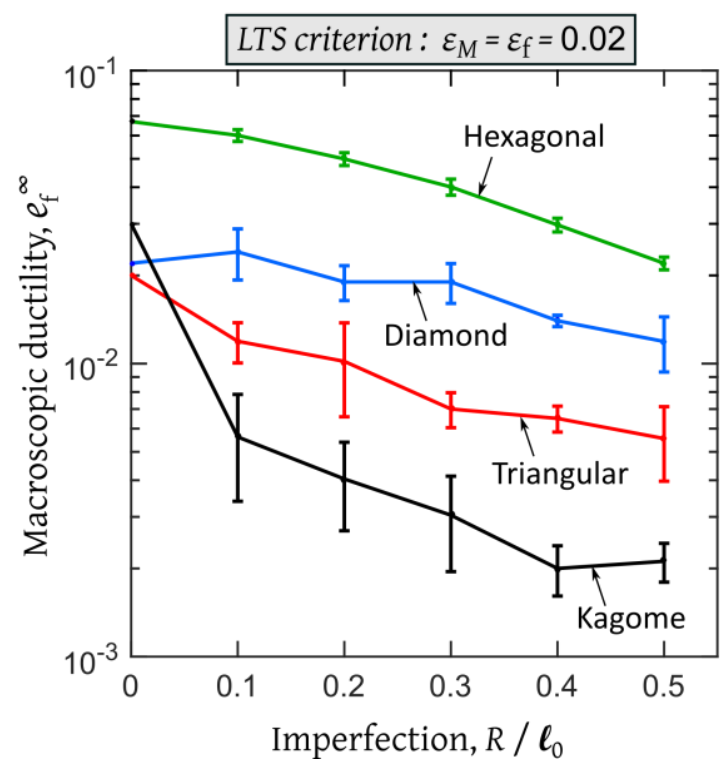

b

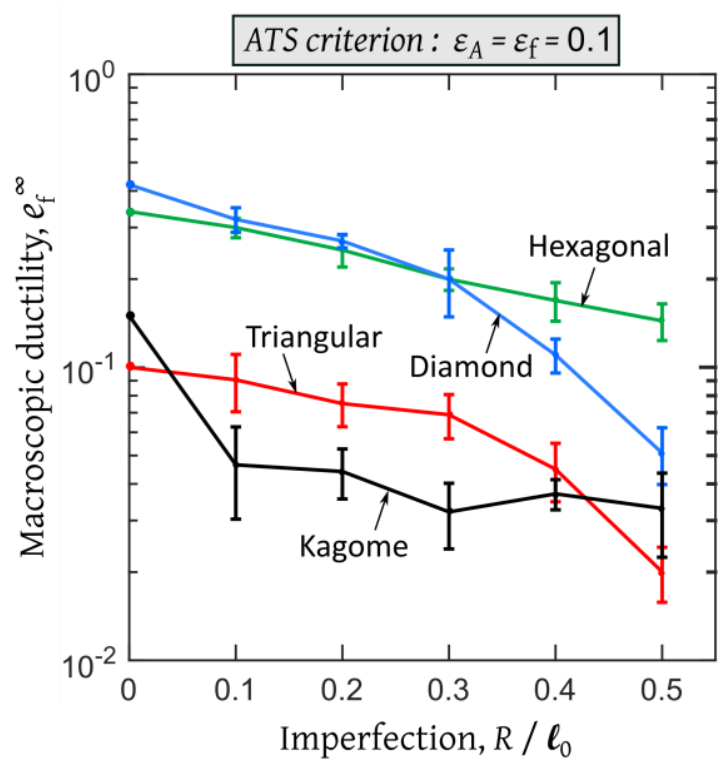

Fig. 11. A comparison of the macroscopic ductility of the four random lattices as a function of the degree of imperfection $R / \ell_{0}$, according to (a) LTS criterion and (b) ATS criterion. The error bars in each case correspond to the standard error for a sample of 10 realisations. 


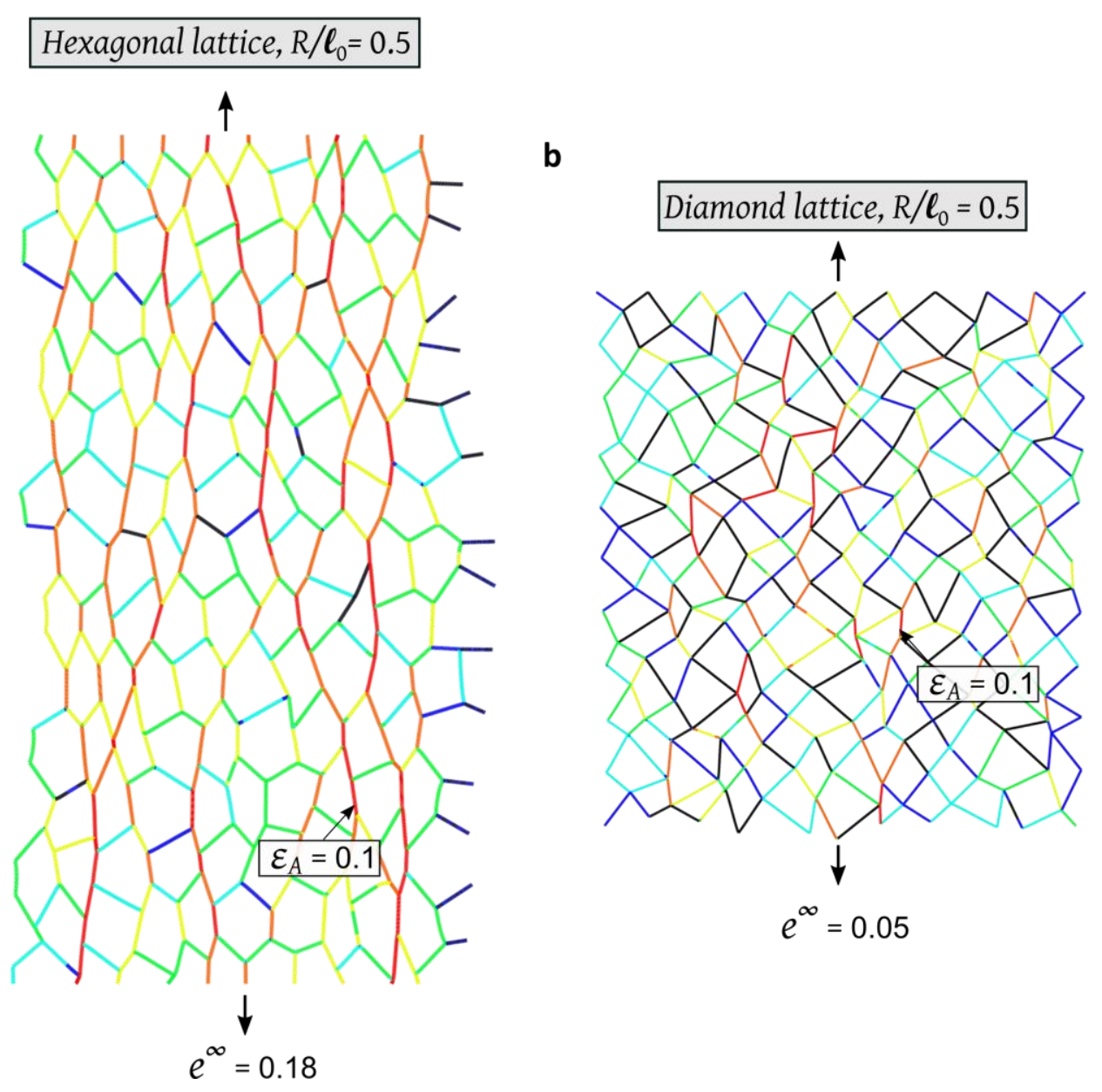

Fig 12. A comparison of the deformed meshes (and contours of axial stress) at the instant when the ATS criterion is met in (a) random hexagonal lattice, and (b) random diamond lattice. For both lattices, $R / \ell_{0}=0.5$. 
a
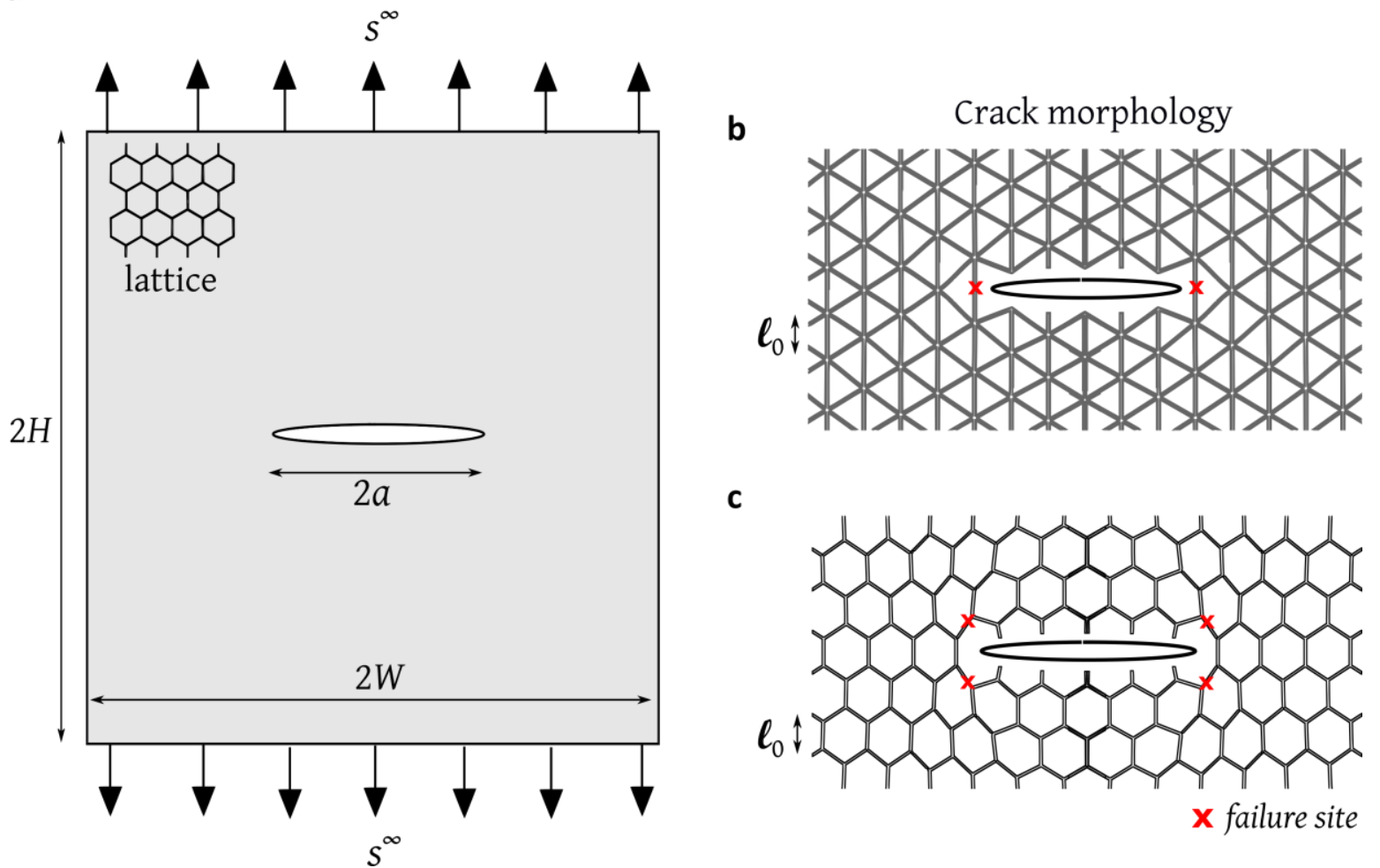

C

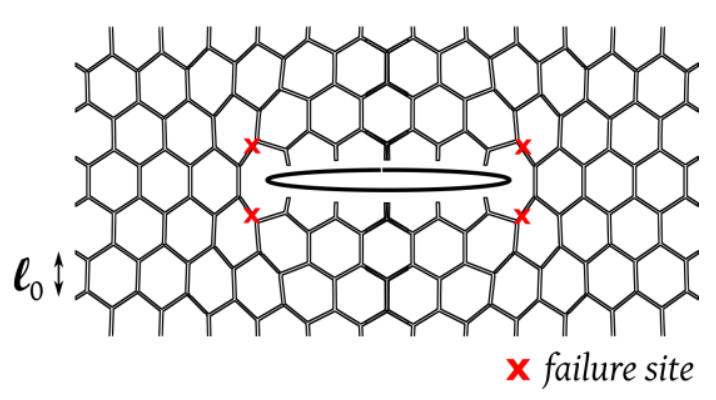

Fig. 13. Centre-cracked plate made from an elastoplastic lattice subjected to uniaxial tension. (a) Geometry and loading; crack morphologies for (b) triangular lattice; and (c) hexagonal lattice. The observed failure site in both the lattices is indicated by $x$ in parts (b) and (c). 
a

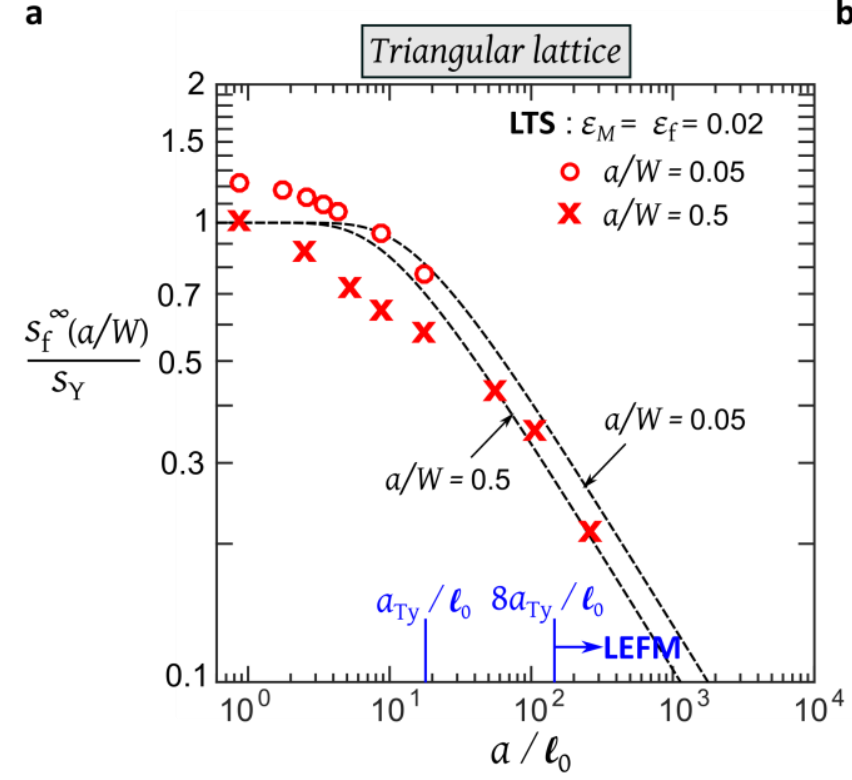

C

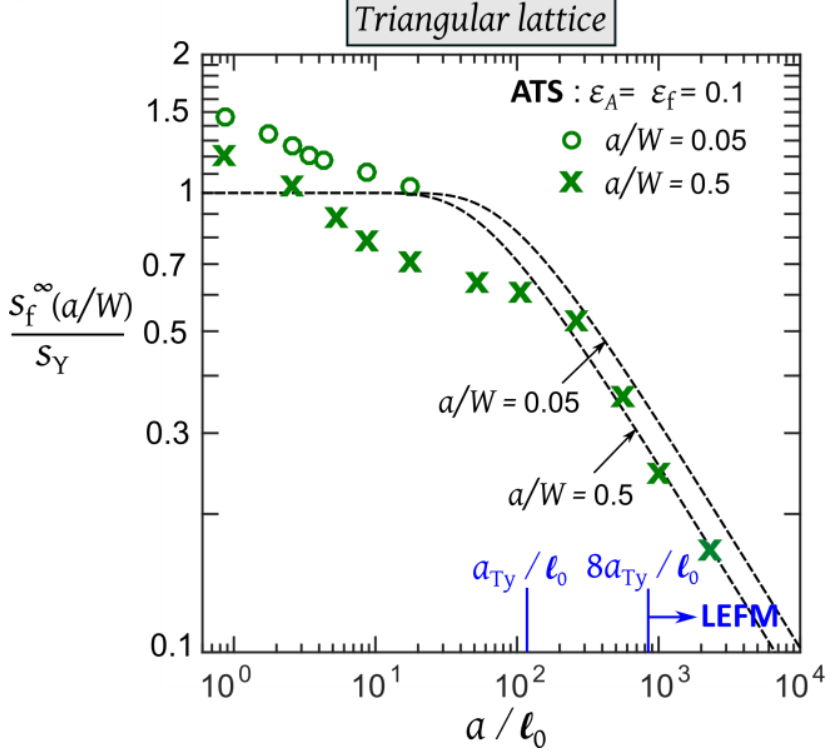

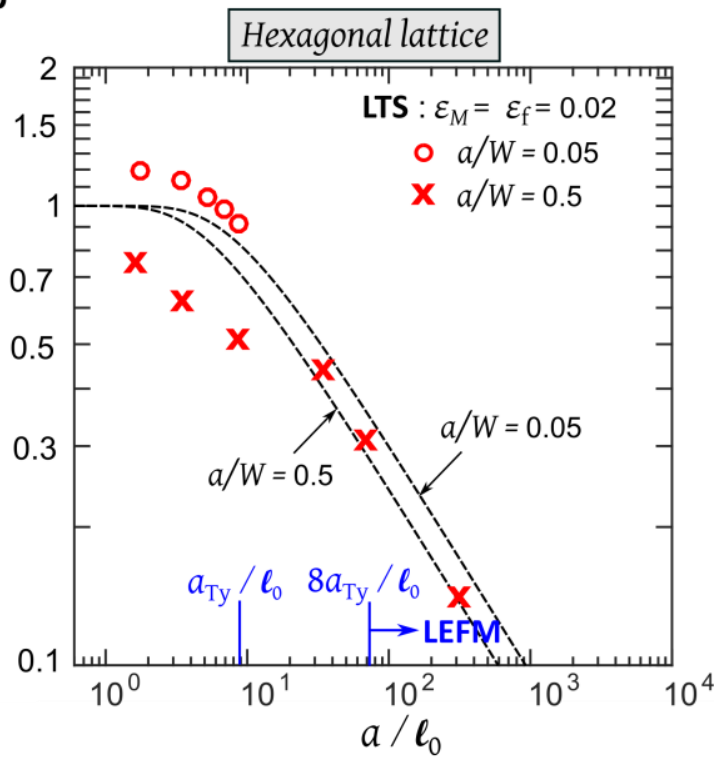

d

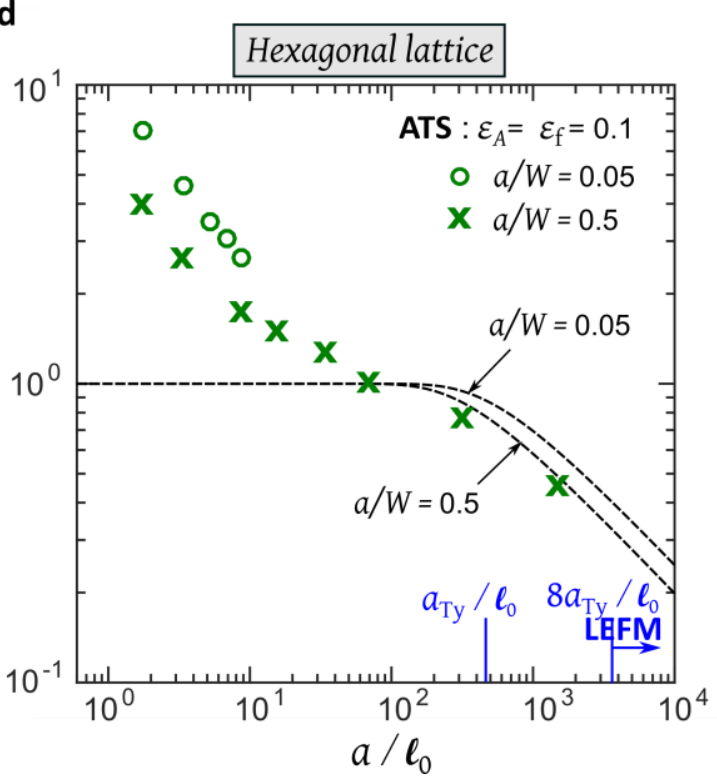

Fig. 14. Notch tensile strength of the elastoplastic lattices as a function of crack length for the choice $\bar{\rho}=0.05$ and $n=10$. (a) triangular lattice with LTS criterion; (b) hexagonal lattice with LTS criterion; (c) triangular lattice with ATS criterion; and (d) hexagonal lattice with ATS criterion. The Dugdale prediction Eq. (21) is shown by dashed lines. 
a

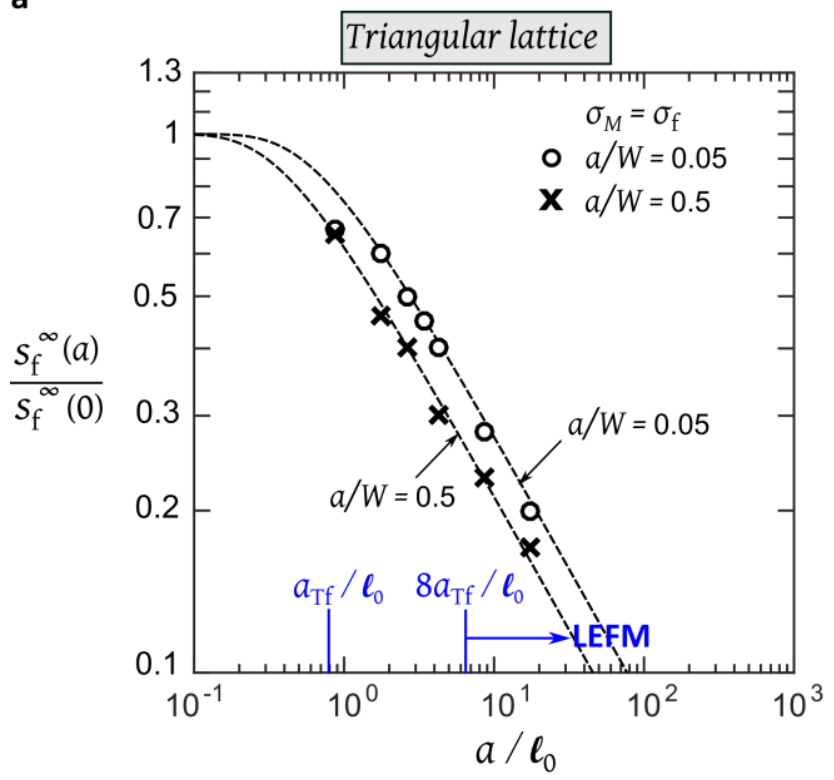

b

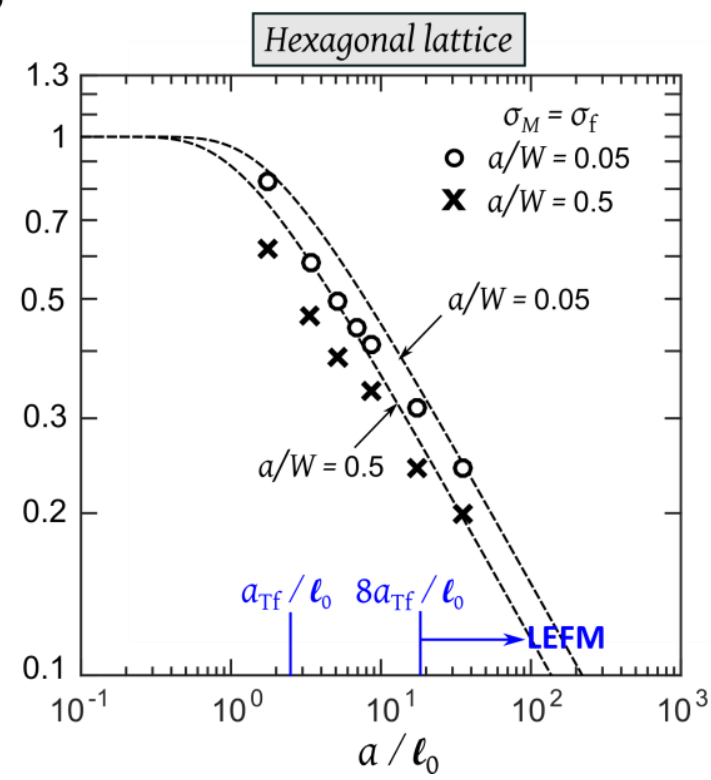

Fig. 15. Notch sensitivity of elastic-brittle lattices: (a) triangular lattice; and (b) hexagonal lattice. The Dugdale prediction, Eq. (24), is shown by dashed lines. 